\title{
Form factor approach to diagonal finite volume matrix elements in Integrable QFT
}

\author{
Balázs Pozsgay ${ }^{1}$ \\ ${ }^{1}$ MTA-BME "Momentum" Statistical Field Theory Research Group \\ 1111 Budapest, Budafoki út 8, Hungary
}

October 29, 2018

\begin{abstract}
We derive an exact formula for finite volume excited state mean values of local operators in $1+1$ dimensional Integrable QFT with diagonal scattering. Our result is a non-trivial generalization of the LeClair-Mussardo series, which is a form factor expansion for finite size ground state mean values.
\end{abstract}

\section{Introduction}

The study of finite volume effects is central to many areas of theoretical physics including Quantum Field Theory (QFT) and Statistical Physics. Understanding the volume dependence of physical observables leads to efficient ways of extracting infinite volume quantities, for example in $3+1$ dimensional lattice QFT calculations. As a first step, one would like to understand how finite volume effects influence the spectrum [1, 2]. As a second step one can also consider composite objects like correlation functions or matrix elements of local operators. An example is given by transition matrix elements in lattice QFT, which were shown to possess a non-trivial volume dependence [3].

Finite size effects are actively investigated in $1+1$ dimensional Integrable QFT, where the integrability of the models allows for an exact determination of physical quantities. For example the exact ground state energy (the Casimir-energy) is known in terms of solutions of certain non-linear integral equations [4, 5, 6, 7, 81. Excited state energies are also known in many cases 19, 10, 11, 12, 13, 14. The techniques developed to study the finite size spectrum of $1+1$ scattering theories found applications and generalizations in the framework of the AdS/CFT correspondence as well [15].

In a Lorentz-invariant $1+1$ dimensional field theory finite size effects are equivalent to finite temperature effects, as can be seen by choosing the compact dimension to lie in the imaginary time direction. Finite temperature correlation functions are relevant to real world condensed matter experiments because the low-energy physics of certain materials leads to effective field theories which are integrable in many cases [16]. This motivated the study of finite temperature correlations in integrable QFT $17,18,19,20,21,22$. In models with diagonal scattering the finite temperature one-point functions are given by the LeClair-Mussardo series [18]. Even though this series has not yet been proven from first principles, it is supported by strong theoretical arguments [23, 20, 21] and numerical checks [24]. On the other hand, an analogous result for the finite temperature two-point function is still missing. The corresponding formula of $\mid 18 \|$ is ill-defined at higher orders and it was criticized in [23, 25]. The leading terms of a well-defined finite temperature expansion were derived in the works [22, 26, 27, 28], but the general pattern of the higher correction terms is not clear yet. 
One way of deriving finite temperature correlations is through a finite volume regularization [29, 20, 21, 26, 22]. In this approach it is essential to know the volume dependence of the finite volume matrix elements of local operators. This problem was solved in 22, 20 to all orders in $1 / L$, where it was shown that the finite volume form factors essentially coincide with the corresponding infinite volume form factors, normalized by the appropriate density of states in rapidity space. Exponential corrections to the asymptotic results of [29, 20] were considered in [30], where the so-called $\mu$-term of the form factors was derived using finite volume bound state quantization (see also [31]).

In the present work we continue the line of research initiated in the works [29, 20, 30]. We consider a subset of the finite volume matrix elements: excited state mean values. Even though these objects are not of direct relevance to finite temperature correlation functions, understanding the structure of the higher exponential corrections in this special case might help to derive results also for the off-diagonal matrix elements, which can lead to a new way of obtaining finite temperature corrections to the two-point function.

The structure of this paper is as follows. In Section 2 we collect the basic results about finite volume QFT which will be used in the subsequent sections. In Section 3 we consider excited states in finite volume and we formulate our basic conjecture for the excited state mean values, which takes the form of a multiple integral series where each integral runs over a non-trivial contour in the complex plain. Sections 4 and 5 include calculations needed to transform this result into a form where each integral runs over the real axis only. In Sections 6 and 7 we calculate our final formulas for the one-particle and two-particle mean values. A general conjecture for higher particle number is given in Section 8. Finally, Section 9 includes our conclusions. The reader who is not interested in the intermediate steps towards our main result in Section 8 (equation (8.2)) may skip sections $4,5,6$ and 7 .

\section{Integrable QFT in finite volume - basic ingredients}

Consider a massive Integrable Quantum Field theory in finite volume $L$ with periodic boundary conditions. The discrete spectrum of the Hamiltonian will be denoted by $|n\rangle_{L}$ with the $n=0$ state being the vacuum. Energy levels are denoted by $E_{n}(L)$. We assume that the energy density of the vacuum is normalized to zero such that $E_{0}(L)$ is the Casimir-energy which decays exponentially with the volume. In the present work we limit ourselves to theories with diagonal scattering. Moreover, for the sake of simplicity we will only consider models with only one particle species. The mass of the single particle will be denoted by $m$.

Let $S(\theta)=e^{i \delta(\theta)}$ be the scattering matrix of the theory (a pure phase in this case). It satisfies the relations

$$
S(\theta) S(-\theta)=1 \quad S(\theta)=S(i \pi-\theta) .
$$

For future use we define the derivative of the phase shift:

$$
\varphi(\theta)=\frac{d}{d \theta}(-i \log S(\theta))
$$

It follows from (2.1) that

$$
\varphi(\theta)=\varphi(-\theta)=\varphi(i \pi+\theta) .
$$

The finite volume ground state energy can be calculated by the Thermodynamic Bethe Ansatz 啫. It is given by

$$
E_{0}(L)=-m \int \frac{d \theta}{2 \pi} \cosh (\theta) \log \left(1+e^{-\varepsilon_{0}(\theta)}\right),
$$

where $\varepsilon_{0}(\theta)$, the so-called pseudoenergy function is given by the solution of the non-linear integral equation

$$
\varepsilon_{0}(\theta)=e(\theta) L-\int_{-\infty}^{\infty} \frac{d \theta^{\prime}}{2 \pi} \varphi\left(\theta-\theta^{\prime}\right) \log \left(1+e^{-\varepsilon_{0}\left(\theta^{\prime}\right)}\right)
$$


with $e(\theta)=m \cosh \theta$ being the one-particle energy and $L$ is the volume.

In this work we are concerned with the finite volume mean values

$$
{ }_{L}\langle n|\mathcal{O}(x)| n\rangle_{L},
$$

where $|n\rangle_{L}$ is an exact eigenstate of the finite volume Hamiltonian normalized to unity and $\mathcal{O}(x)$ is a local operator of the theory, defined with the same normalization as in infinite volume. Translation invariance implies that the mean value does not depend on $x$, therefore this variable will be omitted in the following. For the simplicity we only consider scalar operators.

Our main goal is to derive a form factor expansion for the objects (2.4). The (infinite volume) form factors are defined as the matrix elements of the local operator on infinite volume multiparticle states:

$$
F_{n m}^{\mathcal{O}}\left(\theta_{1}^{\prime}, \ldots, \theta_{n}^{\prime} \mid \theta_{1}, \ldots, \theta_{m}\right)=\left\langle\theta_{1}^{\prime}, \ldots, \theta_{n}^{\prime}|\mathcal{O}| \theta_{1}, \ldots, \theta_{m}\right\rangle
$$

The multiparticle states are equal to the in or out scattering states for a given ordering of the rapidities:

$$
\left|\theta_{1}, \ldots, \theta_{m}\right\rangle= \begin{cases}\left|\theta_{1}, \ldots, \theta_{m}\right\rangle^{\text {in }} & : \theta_{1}>\theta_{2}>\cdots>\theta_{m} \\ \left|\theta_{1}, \ldots, \theta_{m}\right\rangle^{\text {out }} & : \theta_{1}<\theta_{2}<\cdots<\theta_{m}\end{cases}
$$

All form factors can be expressed with the elementary form factors using the crossing relation:

$$
F_{n m}^{\mathcal{O}}\left(\theta_{1}^{\prime}, \ldots, \theta_{n}^{\prime} \mid \theta_{1}, \ldots, \theta_{m}\right)=F_{0, n+m}^{\mathcal{O}}\left(\theta_{1}^{\prime}+i \pi, \ldots, \theta_{n}^{\prime}+i \pi, \theta_{1}, \ldots, \theta_{m}\right) .
$$

The above equation is valid whenever there are no coinciding rapidities, otherwise there are also contact terms present.

In many cases the form factors have been constructed explicitly using the so-called form factor bootstrap program. The idea of this program is to construct all form factor functions which satisfy a certain set of equations (also called the "form factor axioms") and possess certain analyticity properties, and to identify the solutions describing the actual form factors of a given operator. Here we do not review this procedure but instead refer the reader to [32, 33]. We assume that the form factors of the local operator in question are known (or can be calculated in principle). In the calculations presented below we use only two of the general properties, namely the exchange axiom and the kinematical pole property satisfied by the elementary form factors:

$$
\begin{gathered}
F_{n}^{\mathcal{O}}\left(\theta_{1}, \ldots, \theta_{j}, \theta_{j+1}, \ldots, \theta_{n}\right)=S\left(\theta_{j}-\theta_{j+1}\right) F_{n}^{\mathcal{O}}\left(\theta_{1}, \ldots, \theta_{j+1}, \theta_{j}, \ldots, \theta_{n}\right) \\
-i \operatorname{Res}_{\theta=\theta^{\prime}} F_{n+2}^{\mathcal{O}}\left(\theta+i \pi, \theta^{\prime}, \theta_{1}, \ldots, \theta_{n}\right)=\left(1-\prod_{k=1}^{n} S\left(\theta^{\prime}-\theta_{k}\right)\right) F_{n}^{\mathcal{O}}\left(\theta_{1}, \ldots, \theta_{n}\right) .
\end{gathered}
$$

These equations provide the basis for analyzing the diagonal limit of the form factors needed to calculate the finite volume mean values.

\subsection{Previous results for the mean values}

In the case of the ground state mean value it is expected that the large volume limit reproduces the infinite volume expectation value:

$$
\langle\mathcal{O}\rangle_{L}=\langle\mathcal{O}\rangle+\ldots, \quad m L \gg 1
$$

The dots denote finite volume corrections which are of order $e^{-m L}$. An all-order result was found by LeClair and Mussardo in [18] where they considered the equivalent problem of finite 


$$
\langle\mathcal{O}\rangle_{L}=\sum_{n=0}^{\infty} \frac{}{\theta_{1}} \circlearrowright
$$

Figure 1: Graphical interpretation of the LeClair-Mussardo formula for the finite volume ground state expectation values. Time runs in the vertical direction. In the horizontal direction periodic boundary conditions are understood.

temperature one-point functions. They found an infinite integral series (also-called the LeClairMussardo series):

$$
\langle\mathcal{O}\rangle_{L}=\sum_{n} \frac{1}{n !} \int \frac{d \theta_{1}}{2 \pi} \ldots \frac{d \theta_{n}}{2 \pi}\left(\prod_{j} \frac{1}{1+e^{\varepsilon_{0}\left(\theta_{j}\right)}}\right) F_{2 n, c}^{\mathcal{O}}\left(\theta_{1}, \ldots, \theta_{n}\right) .
$$

The form factors entering the integrals are the connected parts of the diagonal infinite volume form factors:

$$
F_{2 n, c}^{\mathcal{O}}\left(\theta_{1} \ldots \theta_{n}\right) \equiv \text { finite piece of } F_{2 n}^{\mathcal{O}}\left(\theta_{1}+i \pi+\eta_{1}, \ldots, \theta_{n}+i \pi+\eta_{n}, \theta_{n}, \ldots, \theta_{1}\right) .
$$

In general the object on the r.h.s. includes terms proportional to $\eta_{j} / \eta_{k}$ and the rule for the connected part is to discard all these terms. For a detailed discussion of this diagonal limit we refer the reader to [20]. Important properties of the functions $F_{2 n, c}^{\mathcal{O}}$ will be discussed in section 占.

The physical interpretation of (2.8) is the following: in the finite volume situation the local operator can interact with an arbitrary number of virtual particles which wind around the finite volume. The amplitude associated to these processes is the properly defined limit of the infinite volume form factor. The fact that the normalization factor associated to these processes is just the product of the weight functions $1 /\left(1+e^{\varepsilon_{0}(\theta)}\right)$ is a highly nontrivial consequence of the integrability of the theory. The volume $L$ only enters (2.8) through the the pseudoenergy $\varepsilon_{0}(\theta)$ which is the unique solution of (2.3). A graphical interpretation of the integral series is shown in Figure 1 .

We note that although (2.8) is generally believed to be true, a rigorous proof from first principles is not yet available. In [21] it was proven to all orders using an expansion for finite volume form factors found in [20] and to be presented below (eq. (2.12)). However, the relation (2.12) itself has not yet been proven (see the discussion below).

Mean values in finite volume excited states have been considered previously in |20|. This work considered the IR limit, when the states can be described with good approximation as Bethe Ansatz states. A first guess for the mean values in this approximation could be that the mean value in a state $\left|\theta_{1}, \ldots, \theta_{K}\right\rangle$ is simply the diagonal form factor $F_{2 K, c}^{\mathcal{O}}\left(\theta_{1}, \ldots, \theta_{K}\right)$, possibly with a normalization factor depending on $L$. However, the situation is more complicated. In |20| it was found that there is expansion for the mean value where each term corresponds to a bipartite partitioning of the rapidities, where one subset of the particles interacts with the operator and the remaining particles only influence the normalization factor associated to this process. 
To be specific, consider the finite volume situation in the limit $m L \gg 1$ and a multiparticle state described by Bethe roots $\left\{\theta_{1}, \ldots, \theta_{K}\right\}$. They satisfy the Bethe equations

$$
Q_{j}=p_{j} L+\sum_{k \neq j} \delta\left(\theta_{j}-\theta_{k}\right)=2 \pi I_{j} \quad j=1 \ldots K, \quad I_{j} \in \mathbb{Z} .
$$

And important quantity is the density of states in rapidity space, which is given by the Jacobian of the mapping given by (2.9):

$$
\rho_{K}\left(\theta_{1}, \ldots, \theta_{K}\right)=\operatorname{det} \mathcal{J}^{j k}, \quad \mathcal{J}^{j k}=\frac{\partial Q_{j}}{\partial \theta_{k}} .
$$

In many non-relativistic models $\rho_{K}$ also describes the norm of the Bethe Ansatz state and it is called the Gaudin determinant [34, 35].

We will also need the minors of the matrix $\mathcal{J}$. For a given bipartite partition

$$
\begin{gathered}
\left\{\theta_{1}, \ldots, \theta_{K}\right\}=\left\{\theta_{+}\right\} \cup\left\{\theta_{-}\right\} \\
\left|\left\{\theta_{+}\right\}\right|=K-n \text { and }\left|\left\{\theta_{-}\right\}\right|=n
\end{gathered}
$$

we define the restricted determinant

$$
\tilde{\rho}_{K-n}\left(\left\{\theta_{+}\right\} \mid\left\{\theta_{-}\right\}\right)=\operatorname{det} \mathcal{J}_{+},
$$

where $\mathcal{J}_{+}$is the sub-matrix of $\mathcal{J}$ corresponding to the particles in the set $\left\{\theta_{+}\right\}$. Note that $\bar{\rho}_{N-n}\left(\left\{\theta_{+}\right\} \mid\left\{\theta_{-}\right\}\right)$still contains information about the complementary set of rapidities $\left\{\theta_{-}\right\}$.

With these notations, the expression for the expectation value reads

$$
\begin{aligned}
\left\langle\theta_{1}, \ldots, \theta_{K}|\mathcal{O}| \theta_{1}, \ldots, \theta_{K}\right\rangle_{L} & = \\
\frac{1}{\rho_{K}\left(\theta_{1}, \ldots, \theta_{K}\right)} & \sum_{\left\{\theta_{+}\right\} \cup\left\{\theta_{-}\right\}} F_{2 n, c}^{\mathcal{O}}\left(\left\{\theta_{-}\right\}\right) \tilde{\rho}_{K-n}\left(\left\{\theta_{+}\right\} \mid\left\{\theta_{-}\right\}\right)+\mathcal{O}\left(e^{-\mu L}\right) .
\end{aligned}
$$

Equation (2.12) is expected to describe the finite size effects to all orders in $1 / L$, beyond which there are only exponentially small corrections. The exponent $\mu$ is a mass scale proportional to $m$ which is determined by the fusion processes in the theory [36. As remarked earlier (2.12) has not yet been proven, but overwhelming numerical evidence has been gathered which support its validity [20, 37, 31]. The only analytical proofs available concern the cases $K=1$ and $K=2$ 20.

\section{$3 \quad$ Excited states}

In many cases there are exact results available about the finite volume energies of the excited states. The first paper to derive TBA equations for particle-like excited states in integrable QFT was [9] where the authors used analytical continuation in the volume parameter $L$ to cross from the ground state to excited states with zero total momentum. This procedure was motivated partly by closely related ideas in Quantum Mechanics [38]. Other works which derived excited state TBA equations include [14, 10, 39]. In the known cases the only difference between the excited state and ground state TBA is the addition of certain source terms, or equivalently a modification of certain integral contours. This property holds also for the various NLIE results, both in field theory and lattice models [11, 12, 13, 40, 11].

Our approach to obtain excited state mean values follows the general idea of the papers [9, 10], namely that there exists an analytic continuation procedure which connects the excited states (or at least a subset of them) to the ground state. Starting from the exact result (2.8) for 
the ground state expectation value it is natural to expect that a proper analytic continuation of the full integral series yields the excited state mean values.

In [9] the analytic continuation was performed in the volume parameter $L$. The volume dependence of the series (2.8) is only through the pseudoenergy function $\varepsilon_{0}(\theta)$ which is the solution of the TBA equation (2.3). In the course of the analytic continuation certain singularities of the pseudoenergy function cross the real line, and this results in a necessary change of integration contour for the TBA equation. We expect that the same holds also for the mean values. Namely, the excited state mean values will be given by equation (2.8) such that $\varepsilon_{0}(\theta)$ has to be replaced by the solution of the appropriate excited state TBA, and the integration contours for the multiple integrals have to be changed accordingly.

In a generic theory a one-particle state is often represented in the excited state TBA as a pair of complex rapidities which are complex conjugates of each other. This is closely related to the fusion processes in the infinite volume theory. Accordingly it was observed in [30] that the leading exponential corrections follow simply from the asymptotic formula (2.12) when single particles are represented by an appropriate finite volume bound state. This pattern of "root doubling" is expected to hold even in the exact result, which would make the formulas quite involved, as already the one-root and two-root problems are technically complicated, as shown below. Therefore in the remainer of this work we only consider the sinh-Gordon model, in which excited state TBA equations of $K$ particles involve exactly $K$ complex roots [39] instead of $2 K$ roots in a typical case in models with particle fusion. However, the analytic continuation procedure we intend to use is not established in the sinh-Gordon model; the paper [39] employed completely different methods to derive the excited state TBA. Therefore the remainder of this paper should be considered as a "technical demonstration" of how the calculations proceed: we just assume that there is a proper analytic continuation and we derive the excited state mean values accordingly. We believe that our results are valid in the sinh-Gordon model and that analogous formulas apply in those cases where single particles are represented by a pair of complex rapidities. We give more remarks on this in the Conclusions.

Turning to the sinh-Gordon model we recall the results of 39. Excited states can be characterized by a set of integer quantum numbers $\left\{I_{1}, I_{2}, \ldots, I_{K}\right\}, K \geq 1$ and a set of real rapidities (Bethe roots) $\left\{\bar{\theta}_{1}, \bar{\theta}_{2}, \ldots, \bar{\theta}_{K}\right\}$. The excited state TBA equation reads

$$
\varepsilon(\theta)=m L \cosh \theta+\sum_{i} \log S\left(\theta-\bar{\theta}_{i}-i \pi / 2\right)-\int \frac{d \theta^{\prime}}{2 \pi} \varphi\left(\theta-\theta^{\prime}\right) \log \left(1+e^{-\varepsilon\left(\theta^{\prime}\right)}\right) .
$$

The condition for the Bethe roots is

$$
\varepsilon\left(\bar{\theta}_{j}+i \pi / 2\right)=i\left(2 I_{j}+1\right) \pi, \quad j=1 \ldots K, \quad I_{j} \in \mathbb{Z} .
$$

The finite size energies are then given by

$$
E=\sum_{j=1}^{K} m \cosh \bar{\theta}_{j}-\int \frac{d \theta}{2 \pi} m \cosh (\theta) \log \left(1+e^{-\varepsilon(\theta)}\right) .
$$

The condition (3.2) can be written explicitly as

$$
m L \sinh \bar{\theta}_{j}+\sum_{k \neq j} \delta\left(\bar{\theta}_{j}-\bar{\theta}_{k}\right)+\int \frac{d \theta^{\prime}}{2 \pi} i \varphi\left(\bar{\theta}_{j}-\theta^{\prime}+i \pi / 2\right) \log \left(1+e^{-\varepsilon\left(\theta^{\prime}\right)}\right)=2 I_{j} \pi .
$$

Equations (3.4) can be interpreted as Bethe equations for the rapidities $\bar{\theta}_{j}$ modified by vacuum polarization effects.

\footnotetext{
${ }^{1}$ We use the notation $\bar{\theta}_{j}$ for the rapidities entering the excited state TBA equations in order to distinguish them from the auxiliary variables $\theta_{j}$ entering the multiple integrals for the mean values.
} 
Given a state $\left|\bar{\theta}_{1}, \ldots, \bar{\theta}_{K}\right\rangle$ we define an integration contour $\mathcal{C}$ which consists of the real line and small circles clockwise around the points $\bar{\theta}_{j}+i \pi / 2$. Then the excited state TBA equations can be re-written as

$$
\begin{gathered}
\varepsilon(\theta)=m L \cosh \theta-\int_{\mathcal{C}} \frac{d \theta^{\prime}}{2 \pi} \varphi\left(\theta-\theta^{\prime}\right) \log \left(1+e^{-\varepsilon\left(\theta^{\prime}\right)}\right) \\
E=-\int_{\mathcal{C}} \frac{d \theta}{2 \pi} m \cosh (\theta) \log \left(1+e^{-\varepsilon(\theta)}\right)
\end{gathered}
$$

where we used the fact that the function $\left(1+e^{-\varepsilon(\theta)}\right)$ has simple zeroes at $\bar{\theta}_{j}+i \pi / 2, j=1 \ldots K$.

As explained above, we conjecture that the mean values of local operators in the given state can be expressed as

$$
\begin{aligned}
&\left\langle\bar{\theta}_{1}, \bar{\theta}_{2}, \ldots, \bar{\theta}_{K}|\mathcal{O}| \bar{\theta}_{1}, \bar{\theta}_{2}, \ldots, \bar{\theta}_{K}\right\rangle_{L}= \\
& \sum_{n} \frac{1}{n !} \int_{\mathcal{C}} \frac{d \theta_{1}}{2 \pi} \ldots \frac{d \theta_{n}}{2 \pi}\left(\prod_{j} \frac{1}{1+e^{\varepsilon\left(\theta_{j}\right)}}\right) F_{2 n, c}^{\mathcal{O}}\left(\theta_{1}, \ldots, \theta_{n}\right),
\end{aligned}
$$

where the pseudoenergy function $\varepsilon(\theta)$ and the roots $\bar{\theta}_{j}$ are given as a solution of the equations (3.1)-(3.2), and the contour $\mathcal{C}$ is determined by the roots $\bar{\theta}_{j}$.

Although the equation above is well-defined and expected to be exact, it is not very enlightening. The remainder of this work is devoted to the evaluation of the residues at the points $\bar{\theta}_{j}+i \pi / 2$ such that in our final formulas the integrals only run over the real line. This leads to a representation where the physical meaning of the individual terms is more transparent.

Note that evaluating all the residues the rapidities $\bar{\theta}_{j}+i \pi / 2$ will appear as multiple insertions in the connected form factors. This motivates the investigation of the degenerate cases for the form factors (section 5). The reader who is not interested in the technical details of these calculations may skip the following four sections and turn to section 8 which presents our final result for arbitrary multiparticle states.

\section{Evaluating the residues}

Here we evaluate the contour integrals in formula (3.7) and express the result as a sum of integrals over the real line. For simplicity we only consider one-particle and two-particle states; it is straightforward to generalize these formulas to higher particle number.

In writing down the multiple integrals we will frequently make use of the shorthand

$$
\int \widetilde{d \theta} \equiv \int \frac{d \theta}{2 \pi} \frac{1}{1+e^{\varepsilon(\theta)}} .
$$

In all formulas below it is understood that $\varepsilon$ is the solution of the TBA equation corresponding to the finite volume state in question.

\subsection{One-particle states}

First we consider one-particle states, ie. $K=1$. In this case the excited state TBA is simply

$$
\varepsilon(\theta)=m L \cosh \theta+\log S(\theta-\bar{\theta}-i \pi / 2)-\int \frac{d \theta^{\prime}}{2 \pi} \varphi\left(\theta-\theta^{\prime}\right) \log \left(1+e^{-\varepsilon\left(\theta^{\prime}\right)}\right) .
$$

We define the function

$$
\bar{Q}(\bar{\theta}, L)=-i \varepsilon(\bar{\theta}+i \pi / 2)=m L \sinh \bar{\theta}-\int \frac{d \theta^{\prime}}{2 \pi} i \varphi\left(\theta^{\prime}-\bar{\theta}+i \pi / 2\right) \log \left(1+e^{-\varepsilon\left(\theta^{\prime}\right)}\right) .
$$


Then the quantization condition for $\bar{\theta}$ is simply $\bar{Q}(\bar{\theta})=2 \pi(I+1 / 2)$.

The integration contour $\mathcal{C}$ in (3.7) consists of the real line and a small circle around the point $\bar{\theta}+i \pi / 2$. The residue of the weight function at this point is

$$
\operatorname{Res}_{\theta=\bar{\theta}+i \pi / 2} \frac{1}{1+e^{\varepsilon(\theta)}}=\left(-\left.\frac{\partial \varepsilon(\theta)}{\partial \theta}\right|_{\theta=\bar{\theta}+i \pi / 2}\right)^{-1} .
$$

Evaluating the residues in the multiple integral series we write

$$
\langle\bar{\theta}|\mathcal{O}| \bar{\theta}\rangle=\sum_{j, k=0}^{\infty} \mathcal{L}_{j k}
$$

Here $\mathcal{L}_{j k}$ represents the contributions where in the $n=j+k$ term in the series we integrate $j$ times around $\bar{\theta}+i \pi / 2$ and $k$ times over the real line. Picking up the residues at $\bar{\theta}+i \pi / 2$ we obtain

$$
\mathcal{L}_{j k}=\frac{1}{j ! k !} \int \widetilde{d \theta_{1}} \ldots \widetilde{d \theta_{k}} \frac{F_{2(j+k), c}^{\mathcal{O}}\left(\bar{\theta}+i \pi / 2, \bar{\theta}+i \pi / 2, \ldots, \theta_{1}, \ldots, \theta_{k}\right)}{n_{1}^{j}},
$$

where

$$
n_{1}=\left.(-i) \frac{\partial \epsilon(\theta)}{\partial \theta}\right|_{\theta=\bar{\theta}+i \pi / 2}=m L \cosh \bar{\theta}+\varphi(0)+\int \frac{d \theta^{\prime}}{2 \pi} i \varphi\left(\theta^{\prime}-\bar{\theta}+i \pi / 2\right) \frac{1}{1+e^{\varepsilon\left(\theta^{\prime}\right)}}\left(\frac{\partial \varepsilon}{\partial \theta}\right),
$$

and in the form factor above there are $j$ insertions of $\bar{\theta}+i \pi / 2$.

It is useful to derive an integral series for the expressions (4.5). Introducing the kernel

$$
\hat{K}\left(\theta, \theta^{\prime}\right)=\varphi\left(\theta-\theta^{\prime}\right) \frac{1}{1+e^{\varepsilon\left(\theta^{\prime}\right)}}
$$

and differentiating (3.4) one obtains

$$
(1-\hat{K}) \frac{\partial \varepsilon}{\partial \theta}=m L \sinh \theta-i \varphi(\theta-\bar{\theta}+i \pi / 2) .
$$

Introducing the resolvent $\hat{M}$ satisfying

$$
(1+\hat{M})(1-\hat{K})=1
$$

we have

$$
\frac{\partial \varepsilon}{\partial \theta}=(1+\hat{M})(m L \sinh \theta-i \varphi(\theta-\bar{\theta}+i \pi / 2)) \quad \frac{\partial \varepsilon}{\partial \bar{\theta}}=(1+\hat{M}) i \varphi(\theta-\bar{\theta}+i \pi / 2) .
$$

Using $1+\hat{M}=\sum_{n=0}^{\infty} K^{n}$ we obtain the integral series

$$
\begin{gathered}
n_{1}=\varphi(0)+m L \cosh \bar{\theta}+\int \widetilde{d \theta} i \varphi(\theta-\bar{\theta}+i \pi / 2)(m L \sinh \theta-i \varphi(\theta-\bar{\theta}+i \pi / 2)) \\
+\sum_{n=2}^{\infty} \int \widetilde{d \theta_{1}} \ldots \widetilde{d \theta_{n}} i \varphi\left(\theta_{1}-\bar{\theta}+i \pi / 2\right) \varphi\left(\theta_{1}-\theta_{2}\right) \ldots \varphi\left(\theta_{n-1}-\theta_{n}\right) \times \\
\times\left(m L \sinh \theta_{n}-i \varphi\left(\theta_{n}-\bar{\theta}+i \pi / 2\right)\right) .
\end{gathered}
$$

The equations (4.3), (4.4) and (4.7) serve as an intermediate result for the one-particle expectation values. Expression (4.4) involves connected diagonal form factors with multiple insertions of the same rapidity. It will be shown in Section that these cases can be expressed as sums of form factors with only a single insertion of $\bar{\theta}+i \pi / 2$. Moreover, after a resummation a remarkably simple formula is found, which reproduces the asymptotic result following from (2.12). This is presented in Section 6 . 


\subsection{Two-particle states}

In the two-particle case the excited state TBA takes the form

$$
\varepsilon(\theta)=m L \cosh \theta+\log S\left(\theta-\bar{\theta}_{1}-i \pi / 2\right)+\log S\left(\theta-\bar{\theta}_{2}-i \pi / 2\right)-\int \frac{d \theta^{\prime}}{2 \pi} \varphi\left(\theta-\theta^{\prime}\right) \log \left(1+e^{-\varepsilon\left(\theta^{\prime}\right)}\right) .
$$

The integration contour $\mathcal{C}$ in (3.7) consists of the real line and two small circles around the points $\bar{\theta}_{1,2}+i \pi / 2$. The residues of the weight function are

$$
\operatorname{Res}_{\theta=\bar{\theta}_{j}+i \pi / 2} \frac{1}{1+e^{\varepsilon(\theta)}}=\left(-\left.\frac{\partial \varepsilon(\theta)}{\partial \theta}\right|_{\theta=\bar{\theta}_{j}+i \pi / 2}\right)^{-1}, \quad j=1,2 .
$$

Taking the derivatives we obtain

$$
\begin{aligned}
n_{j}=-\left.i \frac{\partial \varepsilon(\theta)}{\partial \theta}\right|_{\theta=\bar{\theta}_{j}+i \pi / 2} & =m L \cosh \bar{\theta}_{j}+\varphi\left(\bar{\theta}_{1}-\bar{\theta}_{2}\right)+\varphi(0)- \\
& -\int \frac{d \theta^{\prime}}{2 \pi} i \varphi\left(\theta^{\prime}-\bar{\theta}_{j}-i \pi / 2\right) \frac{1}{1+e^{\varepsilon\left(\theta^{\prime}\right)}} \frac{\partial \varepsilon}{\partial \theta}, \quad j=1,2 .
\end{aligned}
$$

It follows from (4.8) that the derivative of the pseudoenergy satisfies the integral equation

$$
(1-\hat{K}) \frac{\partial \varepsilon}{\partial \theta}=m L \sinh \theta+i \varphi\left(\theta-\bar{\theta}_{1}-i \pi / 2\right)+i \varphi\left(\theta-\bar{\theta}_{2}-i \pi / 2\right)
$$

where the integral operator $K\left(\theta, \theta^{\prime}\right)$ is defined in (4.6). The explicit form of $n_{1,2}$ is

$$
\begin{aligned}
n_{j}= & \varphi(0)+\varphi_{12}+m L \cosh \bar{\theta}_{j} \\
-\int \widetilde{d \theta} i \varphi\left(\theta-\bar{\theta}_{j}-i \pi / 2\right)\left(m L \sinh \theta+i \varphi\left(\theta-\bar{\theta}_{1}-i \pi / 2\right)+i \varphi\left(\theta-\bar{\theta}_{2}-i \pi / 2\right)\right) & \\
-\sum_{n=2}^{\infty} \int \widetilde{d \theta_{1}} \ldots \widetilde{d \theta_{n}} & i \varphi\left(\theta_{1}-\bar{\theta}_{j}-i \pi / 2\right) \varphi\left(\theta_{1}-\theta_{2}\right) \ldots \varphi\left(\theta_{n-1}-\theta_{n}\right) \times \\
& \times\left(m L \sinh \theta_{n}+i \varphi\left(\theta_{n}-\bar{\theta}_{1}-i \pi / 2\right)+i \varphi\left(\theta-\bar{\theta}_{2}-i \pi / 2\right)\right), \quad j=1,2 .
\end{aligned}
$$

Evaluating the residues in (3.7) the result can be written in the form

$$
\left\langle\bar{\theta}_{1}, \bar{\theta}_{2}|\mathcal{O}| \bar{\theta}_{1}, \bar{\theta}_{2}\right\rangle_{L}=\sum_{j, k, l} \mathcal{L}_{j k l}
$$

where $\mathcal{L}_{j k l}$ denotes those contributions of the $n=j+k+l$ term of the original series where $\bar{\theta}_{1}+i \pi / 2$ has been inserted $j$ times, $\bar{\theta}_{2}+i \pi / 2$ has been inserted $k$ times, and there are $l$ auxiliary rapidities which are integrated over. Explicitly

$\mathcal{L}_{j k l}=\frac{1}{j ! k ! l !} \int \widetilde{d \theta_{1}} \ldots \widetilde{d \theta_{l}} \frac{F_{2(j+k+l), c}^{\mathcal{O}}\left(\bar{\theta}_{1}+i \pi / 2, \bar{\theta}_{1}+i \pi / 2, \ldots, \bar{\theta}_{2}+i \pi / 2, \bar{\theta}_{2}+i \pi / 2, \ldots, \theta_{1}, \ldots, \theta_{l}\right)}{n_{1}^{j} n_{2}^{k}}$.

Equations (4.12), (4.13) together with (4.9) represent an explicit result for the two-particle mean values. However, similar to the one-particle case further investigation of the degenerate cases of the form factors leads to a remarkably simpler formula, presented in Section 0. Eq. (4.13) motivates the study of those diagonal form factors, where there are multiple insertions of two different rapidities. 


\section{Properties of the connected diagonal form factors}

In this section we consider the connected evaluation of the diagonal form factors of a local operator $\mathcal{O}$. They are defined as

$$
F_{2 n, c}^{\mathcal{O}}\left(\theta_{1} \ldots \theta_{n}\right)=\text { finite piece of } F_{2 n}^{\mathcal{O}}\left(\theta_{1}+i \pi+\eta_{1}, \ldots, \theta_{n}+i \pi+\eta_{n}, \theta_{n}, \ldots, \theta_{1}\right) .
$$

It follows from the exchange axiom (2.6) that the functions above are completely symmetric in their variables. They are meromorphic functions on the entire complex plain and are invariant with respect to an overall boost of the rapidities. It can be proven that they are $i \pi$ periodic and that they inherit the clustering property: 42]

$$
\lim _{\Lambda \rightarrow \infty} F_{2 n+2 m, c}^{\mathcal{O}}\left(\theta_{1}+\Lambda, \ldots, \theta_{n}+\Lambda, \theta_{1}^{\prime}, \ldots, \theta_{m}^{\prime}\right)=\frac{1}{\langle\mathcal{O}\rangle} F_{2 n, c}^{\mathcal{O}}\left(\theta_{1}, \ldots, \theta_{n}\right) F_{2 m, c}^{\mathcal{O}}\left(\theta_{1}^{\prime}, \ldots, \theta_{m}^{\prime}\right) .
$$

No theorems are known about the growth properties of the diagonal form factors. However we can assume that there exists a $K \in \mathbb{R}^{+}$such that for any $n$

$$
\left|F_{2 n, c}^{\mathcal{O}}\left(\theta_{1}, \ldots, \theta_{n}\right)\right|<n ! K^{n}, \quad \theta_{j} \in \mathbb{C} .
$$

This is in accordance with all previous experience and is enough to ensure the convergence of the series (2.8) for large enough $L$.

We note that the two-particle connected form factor $F_{2 c}^{\mathcal{O}}(\theta)$ does not depend on $\theta$ by Lorentzinvariance. Therefore it will be simply denoted by $F_{2 c}^{\mathcal{O}}$ in the rest of this work.

\subsection{Degenerate cases}

In the following we consider degenerate diagonal form factors, ie. when there are multiple copies of the same rapidity present. We only consider those cases which are relevant for the one-particle and two-particle mean values, namely when there are multiple copies of at most two different rapidities.

In the calculations we will extensively make use of sets and lists of indices, therefore it is useful to introduce a few definitions and notations for further use.

A multiset is a generalization of a set allowing members to appear more than once. The difference between a multiset and a sequence is that in a multiset the order of the elements does not matter, whereas in a sequence it does. Multisets of integers will be denoted using curly braces, for example $A=\left\{a_{1}, a_{2}, \ldots, a_{n}\right\}$. Sequences of integers will be denoted by braces: $s=\left(s_{1}, s_{2}, \ldots, s_{n}\right)$. If the explicit numbers are given then we don't use separation marks, for example $s=(1342)$. Both for sequences and multisets, multiple addition of a given number will be sometimes denoted in the superscript, for example

$$
\left\{1^{(\times 3)}\right\} \equiv\{1,1,1\} .
$$

Concatenation of sequences or addition of new elements will be denoted simply by writing down the constituents without separation marks. For example if

$$
A=(123) \quad \text { and } \quad B=(31)
$$

then

$$
(2 A 1 B)=(2123131)
$$

Unions of multisets is defined as a complete addition of the elements and it will be denoted simply by comma, for example if $A=\{1,1\}$ and $B=\{1,2,3\}$ then

$$
\{A, B\}=\{1,1,1,2,3\} \text {. }
$$


The diagonal form factor evaluated on a multiset $A$ is defined as

$$
F_{2 n, c}^{\mathcal{O}}(A) \equiv F_{2 n, c}^{\mathcal{O}}\left(\theta_{A_{1}}, \theta_{A_{2}}, \ldots, \theta_{A_{n}}\right) .
$$

Given a sequence of integers $s$ we define

$$
[s]_{\varphi} \equiv \varphi_{s_{1} s_{2}} \varphi_{s_{2} s_{3}} \ldots \varphi_{s_{n-1} s_{n}}
$$

where it is understood that $n$ is the length of $s$ and

$$
\varphi_{j k}=\varphi\left(\theta_{j}-\theta_{k}\right)
$$

In the simplest case $[11]_{\varphi}=\varphi(0)$. Sometimes we will use the shorthand $\varphi_{0}=\varphi(0)$.

Given a multiset $A$ we define $S_{a, b}(A)$ to be the set of all sequences, which contain every member of $A$ exactly once, and where the first and last elements are $a$ and $b$, respectively. It is understood that $S_{a, b}(A)$ is empty when $a \notin A$ or $b \notin A$. For example

$$
S_{1,2}(\{1,1,2,3\})=\{(1132),(1312)\} .
$$

\subsubsection{Multiple copies of one rapidity}

The simplest degenerate case is when in the four-particle diagonal form factor the two rapidities coincide.

\section{Theorem 1.}

$$
F_{4 c}^{\mathcal{O}}(\theta, \theta)=2 \varphi_{0} F_{2 c}^{\mathcal{O}}
$$

Proof. Consider the form factor $F_{4}^{\mathcal{O}}\left(\theta_{1}, \theta_{2}, i \pi+\theta_{3}, i \pi+\theta_{4}\right)$ at $\theta_{1,2,3,4} \rightarrow \theta$. In this case there are 4 kinematical poles and no double singularities. Subtracting all four poles we obtain the fully connected form factor 2

$$
\begin{aligned}
F_{4, f c}^{\mathcal{O}}= & F_{4}^{\mathcal{O}}\left(\theta_{1}+i \pi, \theta_{2}+i \pi, \theta_{3}, \theta_{4}\right)- \\
& -\frac{i}{\theta_{1}-\theta_{3}}\left[S\left(\theta_{1}-\theta_{2}\right)-S\left(\theta_{3}-\theta_{4}\right)\right] F_{2}^{\mathcal{O}}\left(\theta_{2}+i \pi, \theta_{4}\right) \\
& -\frac{i}{\theta_{2}-\theta_{3}}\left[1-S\left(\theta_{3}-\theta_{4}\right) S\left(\theta_{1}-\theta_{2}\right)\right] F_{2}^{\mathcal{O}}\left(\theta_{1}+i \pi, \theta_{4}\right) \\
& -\frac{i}{\theta_{1}-\theta_{4}}\left[S\left(\theta_{1}-\theta_{2}\right) S\left(\theta_{3}-\theta_{4}\right)-1\right] F_{2}^{\mathcal{O}}\left(\theta_{2}+i \pi, \theta_{3}\right) \\
& -\frac{i}{\theta_{2}-\theta_{4}}\left[S\left(\theta_{3}-\theta_{4}\right)-S\left(\theta_{1}-\theta_{2}\right)\right] F_{2}^{\mathcal{O}}\left(\theta_{1}+i \pi, \theta_{3}\right) .
\end{aligned}
$$

The object above has manifestly the same exchange properties as the original form factor. Therefore it vanishes as $\theta_{1} \rightarrow \theta_{2}$ or $\theta_{3} \rightarrow \theta_{4}$ and even the point $\theta_{1}=\theta_{2}=\theta_{3}=\theta_{4}$ is completely regular and continuous. Using the approximation $S(\varepsilon) \approx-1-i \varphi_{0} \varepsilon$ we obtain

$$
\begin{aligned}
F_{4, f c}^{\mathcal{O}} \approx & F_{4}^{\mathcal{O}}\left(\theta_{1}+i \pi, \theta_{2}+i \pi, \theta_{3}, \theta_{4}\right)- \\
& -\frac{1}{\theta_{1}-\theta_{3}}\left(\theta_{1}-\theta_{2}-\theta_{3}+\theta_{4}\right) \varphi_{0} F_{2}^{\mathcal{O}}\left(\theta_{2}+i \pi, \theta_{4}\right) \\
& -\frac{1}{\theta_{2}-\theta_{3}}\left(\theta_{3}-\theta_{4}+\theta_{1}-\theta_{2}\right) \varphi_{0} F_{2}^{\mathcal{O}}\left(\theta_{1}+i \pi, \theta_{4}\right) \\
& -\frac{1}{\theta_{1}-\theta_{4}}\left(-\theta_{1}+\theta_{2}-\theta_{3}+\theta_{4}\right) \varphi_{0} F_{2}^{\mathcal{O}}\left(\theta_{2}+i \pi, \theta_{3}\right) \\
& -\frac{1}{\theta_{2}-\theta_{4}}\left(\theta_{3}-\theta_{4}-\theta_{1}+\theta_{2}\right) \varphi_{0} F_{2}^{\mathcal{O}}\left(\theta_{1}+i \pi, \theta_{3}\right) .
\end{aligned}
$$

\footnotetext{
${ }^{2}$ The main idea of this proof was suggested by Gábor Takács.
} 
The connected FF is obtained by taking $\theta_{1} \rightarrow \theta_{4}$ and $\theta_{2} \rightarrow \theta_{3}$ and subtracting the poles of the form $\left(\theta_{1}-\theta_{4}\right) /\left(\theta_{2}-\theta_{3}\right)$ and $\left(\theta_{2}-\theta_{3}\right) /\left(\theta_{1}-\theta_{4}\right)$ :

$$
\begin{aligned}
F_{4 c}^{\mathcal{O}}\left(\theta_{1}, \theta_{2}\right)= & \lim _{\theta_{1} \rightarrow \theta_{4}} \lim _{\theta_{2} \rightarrow \theta_{3}}\left[F_{4}^{\mathcal{O}}\left(\theta_{1}+i \pi, \theta_{2}+i \pi, \theta_{3}, \theta_{4}\right)-\right. \\
& \left.-\frac{\theta_{1}-\theta_{4}}{\theta_{2}-\theta_{3}} \varphi_{0} F_{2}^{\mathcal{O}}\left(\theta_{1}+i \pi, \theta_{4}\right)-\frac{\theta_{2}-\theta_{3}}{\theta_{1}-\theta_{4}} \varphi_{0} F_{2}^{\mathcal{O}}\left(\theta_{2}+i \pi, \theta_{3}\right)\right] .
\end{aligned}
$$

Using

$$
\lim _{\theta_{1,2,3,4} \rightarrow \theta} F_{4, f c}^{\mathcal{O}}\left(\theta_{1}+i \pi, \theta_{2}+i \pi, \theta_{3}, \theta_{4}\right)=0
$$

we obtain

$$
\lim _{\theta_{1} \rightarrow \theta_{2}} F_{4 c}^{\mathcal{O}}\left(\theta_{1}, \theta_{2}\right)=2 \varphi_{0} F_{2, c}^{\mathcal{O}}
$$

A similar calculation can be performed in the three-particle case when two rapidities coincide:

\section{Theorem 2.}

$$
F_{6 c}^{\mathcal{O}}\left(\theta_{1}, \theta_{1}, \theta_{3}\right)=2\left(\varphi_{0} F_{4 c}^{\mathcal{O}}\left(\theta_{1}, \theta_{3}\right)+\varphi_{13}^{2} F_{2 c}^{\mathcal{O}}\right)
$$

Proof. Consider the fully connected form factor

$$
\begin{aligned}
F_{6, f c}^{\mathcal{O}}= & F_{6}^{\mathcal{O}}\left(\theta_{1}+i \pi, \theta_{2}+i \pi, \theta_{3}+i \pi, \theta_{3}^{\prime}, \theta_{2}^{\prime}, \theta_{1}^{\prime}\right)- \\
& -\frac{i}{\theta_{1}-\theta_{1}^{\prime}}\left[S\left(\theta_{1}-\theta_{2}\right) S\left(\theta_{1}-\theta_{3}\right) S\left(\theta_{2}^{\prime}-\theta_{1}^{\prime}\right) S\left(\theta_{3}^{\prime}-\theta_{1}^{\prime}\right)-1\right] F_{4}^{\mathcal{O}}\left(\theta_{2}+i \pi, \theta_{3}+i \pi, \theta_{3}^{\prime}, \theta_{2}^{\prime}\right) \\
& -\frac{i}{\theta_{1}-\theta_{2}^{\prime}}\left[S\left(\theta_{1}-\theta_{2}\right) S\left(\theta_{1}-\theta_{3}\right) S\left(\theta_{3}^{\prime}-\theta_{2}^{\prime}\right)-S\left(\theta_{2}^{\prime}-\theta_{1}^{\prime}\right)\right] F_{4}^{\mathcal{O}}\left(\theta_{2}+i \pi, \theta_{3}+i \pi, \theta_{3}^{\prime}, \theta_{1}^{\prime}\right) \\
& -\frac{i}{\theta_{2}-\theta_{1}^{\prime}}\left[S\left(\theta_{2}-\theta_{3}\right) S\left(\theta_{2}^{\prime}-\theta_{1}^{\prime}\right) S\left(\theta_{3}^{\prime}-\theta_{1}^{\prime}\right)-S\left(\theta_{1}-\theta_{2}\right)\right] F_{4}^{\mathcal{O}}\left(\theta_{1}+i \pi, \theta_{3}+i \pi, \theta_{3}^{\prime}, \theta_{2}^{\prime}\right) \\
& -\frac{i}{\theta_{2}-\theta_{2}^{\prime}}\left[S\left(\theta_{2}-\theta_{3}\right) S\left(\theta_{3}^{\prime}-\theta_{2}^{\prime}\right)-S\left(\theta_{1}-\theta_{2}\right) S\left(\theta_{2}^{\prime}-\theta_{1}^{\prime}\right)\right] F_{4}^{\mathcal{O}}\left(\theta_{1}+i \pi, \theta_{3}+i \pi, \theta_{3}^{\prime}, \theta_{1}^{\prime}\right) .
\end{aligned}
$$

This object satisfies the exchange axioms and therefore it vanishes at the degenerate point.

Expanding the pre-factors to first order

$$
\begin{aligned}
F_{6, f c}^{\mathcal{O}}= & F_{6}^{\mathcal{O}}\left(\theta_{1}+i \pi, \theta_{2}+i \pi, \theta_{3}+i \pi, \theta_{3}^{\prime}, \theta_{2}^{\prime}, \theta_{1}^{\prime}\right) \\
& +\frac{1}{\varepsilon_{1}}\left[\varphi_{0}\left(\varepsilon_{1}-\varepsilon_{2}\right)+\varphi_{13}\left(\varepsilon_{1}-\varepsilon_{3}\right)\right] F_{4}^{\mathcal{O}}\left(\theta_{2}+i \pi, \theta_{3}+i \pi, \theta_{3}^{\prime}, \theta_{2}^{\prime}\right) \\
& -\frac{1}{\theta_{1}-\theta_{2}^{\prime}}\left[\varphi_{13}\left(\theta_{1}-\theta_{2}^{\prime}-\varepsilon_{3}\right)+\varphi_{0}\left(\theta_{1}+\theta_{1}^{\prime}-\theta_{2}-\theta_{2}^{\prime}\right)\right] F_{4}^{\mathcal{O}}\left(\theta_{2}+i \pi, \theta_{3}+i \pi, \theta_{3}^{\prime}, \theta_{1}^{\prime}\right) \\
& -\frac{1}{\theta_{2}-\theta_{1}^{\prime}}\left[\varphi_{13}\left(\theta_{2}-\theta_{1}^{\prime}-\varepsilon_{3}\right)-\varphi_{0}\left(\theta_{1}+\theta_{1}^{\prime}-\theta_{2}-\theta_{2}^{\prime}\right)\right] F_{4}^{\mathcal{O}}\left(\theta_{1}+i \pi, \theta_{3}+i \pi, \theta_{3}^{\prime}, \theta_{2}^{\prime}\right) \\
& +\frac{1}{\varepsilon_{2}}\left[\varphi_{13}\left(\varepsilon_{2}-\varepsilon_{3}\right)-\varphi_{0}\left(\varepsilon_{1}-\varepsilon_{2}\right)\right] F_{4}^{\mathcal{O}}\left(\theta_{1}+i \pi, \theta_{3}+i \pi, \theta_{3}^{\prime}, \theta_{1}^{\prime}\right) .
\end{aligned}
$$

We have to collect all those contributions which are non-singular in the limit $\varepsilon_{1,2,3} \rightarrow 0$. From the first and last line we get (already assuming $\theta_{2} \rightarrow \theta_{1}$ )

$$
2\left(\varphi_{0}+\varphi_{13}\right) F_{4 c}^{\mathcal{O}}\left(\theta_{1}, \theta_{3}\right) \text {. }
$$

In the second and third line we use

$$
\begin{aligned}
& F_{4}^{\mathcal{O}}\left(\theta_{2}+i \pi, \theta_{3}+i \pi, \theta_{3}^{\prime}, \theta_{1}^{\prime}\right)=F_{4 c}^{\mathcal{O}}\left(\theta_{1}, \theta_{3}\right)+\left(\frac{\theta_{2}-\theta_{1}^{\prime}}{\varepsilon_{3}}+\frac{\varepsilon_{3}}{\theta_{2}-\theta_{1}^{\prime}}\right) \varphi_{13} F_{2 c}^{\mathcal{O}} \\
& F_{4}^{\mathcal{O}}\left(\theta_{1}+i \pi, \theta_{3}+i \pi, \theta_{3}^{\prime}, \theta_{2}^{\prime}\right)=F_{4 c}^{\mathcal{O}}\left(\theta_{1}, \theta_{3}\right)+\left(\frac{\theta_{1}-\theta_{2}^{\prime}}{\varepsilon_{3}}+\frac{\varepsilon_{3}}{\theta_{1}-\theta_{2}^{\prime}}\right) \varphi_{13} F_{2 c}^{\mathcal{O}} .
\end{aligned}
$$


This way we obtain the contributions

$$
-\left(2 \varphi_{13}+2 \varphi_{0}-\varphi_{0}\left(\frac{\theta_{2}-\theta_{1}^{\prime}}{\theta_{1}-\theta_{2}^{\prime}}+\frac{\theta_{1}-\theta_{2}^{\prime}}{\theta_{2}-\theta_{1}^{\prime}}\right)\right) F_{4 c}^{\mathcal{O}}\left(\theta_{1}, \theta_{3}\right)+\left(\frac{\theta_{2}-\theta_{1}^{\prime}}{\theta_{1}-\theta_{2}^{\prime}}+\frac{\theta_{1}-\theta_{2}^{\prime}}{\theta_{2}-\theta_{1}^{\prime}}\right) \varphi_{13}^{2} F_{2 c}^{\mathcal{O}} .
$$

Taking $\theta_{1}^{\prime} \rightarrow \theta_{1}$ and $\theta_{2}^{\prime} \rightarrow \theta_{2}$

$$
-\left(2 \varphi_{13}+4 \varphi_{0}\right) F_{4 c}^{\mathcal{O}}\left(\theta_{1}, \theta_{3}\right)-2 \varphi_{13}^{2} F_{2 c}^{\mathcal{O}}
$$

Adding all the contributions we obtain the statement of the theorem.

The higher particle case of two coinciding rapidities is given as follows.

Theorem 3. Let $A=\{2,3, \ldots, m\}$. Then

$$
F_{c}^{\mathcal{O}}(1,1, A)=2 \sum_{A=A^{+} \cup A^{-}} F_{c}^{\mathcal{O}}\left(1, A^{+}\right) \sum_{s \in S_{1,1}\left(1,1, A^{-}\right)}[s]_{\varphi}
$$

The sum in (5.3) runs over all bi-partite partitions of the set A. For example in the case of $A=\{2,3\}$ we have

$$
\begin{aligned}
F_{8 c}^{\mathcal{O}}(1,1,2,3)=2( & F_{6 c}^{\mathcal{O}}(1,2,3)[11]_{\varphi}+ \\
& F_{4 c}^{\mathcal{O}}(1,2)[131]_{\varphi}+F_{4 c}^{\mathcal{O}}(1,3)[121]_{\varphi}+ \\
& \left.F_{2 c}^{\mathcal{O}}(1)\left([1231]_{\varphi}+[1321]_{\varphi}\right)\right)
\end{aligned}
$$

Proof. The proof can be given following the same steps as in the three-particle case presented above.

The following theorem concerns the case when there are more than two occurrences of $\theta_{1}$ in the form factor:

Theorem 4. Let $B=\left\{1^{(\times n)}\right\}$ with $n \geq 2$ and $A$ an arbitrary multiset not including 1 . Then

$$
F_{2(n+m), c}^{\mathcal{O}}(B, A)=n ! \sum_{A=A^{+} \cup A^{-}} F_{c}^{\mathcal{O}}\left(1, A^{+}\right) \sum_{s \in S_{1,1}\left(B, A^{-}\right)}[s]_{\varphi}
$$

where $|A|=m$.

Proof. For technical reasons introduce new labels to the first $n$ rapidities as $\left(1_{a}, 1_{b}, \ldots\right)$. The degenerate form factor can be obtained by repeated use of eq. (5.3). Here for technical reasons we distinguish the first rapidities too, therefore (5.3) can be written down without a factor of 2 , extending the summation over all possible paths starting and ending with either of the 1's. Then the repeated use of Theorem 3 results in all possible paths starting and ending with one of the 1 's. Removing the labels at the end results in a factor of $n$ !.

As a special case of the above theorem we obtain the result for the completely degenerate case:

$$
F_{2 n, c}^{\mathcal{O}}(1,1, \ldots, 1)=n ! \varphi(0)^{n-1} F_{2, c}^{\mathcal{O}}
$$




\subsubsection{Multiple copies of two rapidities}

Theorem 5. Let $A=\left\{1^{(\times n)}, 2^{(\times m)}\right\}$ with $n, m \geq 2$. Then

$$
F_{2(n+m), c}^{\mathcal{O}}(A)=n ! m !\left(\frac{F_{4 c}^{\mathcal{O}}(1,2)}{\varphi_{12}} \sum_{s \in S_{1,2}(A)}[s]_{\varphi}+F_{2 c}^{\mathcal{O}}(1) \sum_{s \in S_{1,1}(A)}[s]_{\varphi}+F_{2 c}^{\mathcal{O}}(2) \sum_{s \in S_{2,2}(A)}[s]_{\varphi}\right) .
$$

Proof. There are two ways to calculate this diagonal case. We can apply Theorem $⿴$ first to the 1 's, then to the 2's, or the other way around. Performing the steps in the first way, it is easy to see that the only contributions to the coefficient of $F_{2 c}^{\mathcal{O}}(1)$ are those given above. The coefficient of $F_{2 c}^{\mathcal{O}}(2)$ can be obtained by performing the two steps in the second way.

In order to obtain the coefficient of $F_{4 c}^{\mathcal{O}}(1,2)$ we attach extra labels to the numbers as $\left\{1_{a}, 1_{b}, \ldots, 2_{a}, 2_{b}, \ldots\right\}$. We obtain terms of the form $[1 B 1]_{\varphi}[2 C 2]_{\varphi}$, where $A=\{1,1,2,2, B, C\}$ and $C$ consists only of 2's ( $B$ may include both 1's and 2 's) and terms of the form $[1 B 1]_{\varphi}$ such that $A=\{1,1,2, B\}$. For the terms in the first case we use the identity

$$
[1 B 1]_{\varphi}[2 C 2]_{\varphi}=\frac{[1 B 12 C 2]_{\varphi}}{\varphi_{12}}
$$

whereas in the second case we have

$$
[1 B 1]_{\varphi}=\frac{[1 B 12]_{\varphi}}{\varphi_{12}}
$$

This way we obtain a summation over all paths starting with one of the 1's and ending with one of the 2's. Finally removing the labels we obtain a factor of $n ! m !$.

Finally we consider the case when there are a multiple 1's and 2's and an arbitrary number of other rapidities.

Theorem 6. Let $A=\left\{1^{(\times n)}, 2^{(\times m)}, B\right\}$ with $B=\{3,4, \ldots, k+2\}$ such that $|B|=k$. The degenerate form factor is equal to

$$
\begin{aligned}
F_{2(n+m+o), c}^{\mathcal{O}}(A)=n ! m ! & \sum_{B=B^{+} \cup B^{-}} \\
& \left(\frac{F_{c}^{\mathcal{O}}\left(1,2, B^{+}\right)}{\varphi_{12}} \sum_{s \in \tilde{S}_{1,2}\left(A \backslash B^{+}\right)}[s]_{\varphi}+\right. \\
& \left.+F_{c}^{\mathcal{O}}\left(1, B^{+}\right) \sum_{s \in S_{1,1}\left(A \backslash B^{+}\right)}[s]_{\varphi}+F_{c}^{\mathcal{O}}\left(2, B^{+}\right) \sum_{s \in S_{2,2}\left(A \backslash B^{+}\right)}[s]_{\varphi}\right),
\end{aligned}
$$

where $\tilde{S}_{1,2}(A)$ is the sum of all paths consisting of the elements of $A$, which start with 1, end with 2, and the first number after the rightmost 1 is 2. For example

$$
\tilde{S}_{1,2}(\{1,1,2,2,3\})=\{(12312),(13122),(13212),(11232)\} .
$$

Proof. The theorem can be proven along the lines of the previous proof. The coefficients of the form factors $F_{c}^{\mathcal{O}}\left(1, B^{+}\right)$and $F_{c}^{\mathcal{O}}\left(2, B^{+}\right)$are easily obtained by performing the steps of theorem 1 first for the 1 's, then for the 2 's, or the other way around, respectively.

In order to obtain the coefficients of the form factors $F_{c}^{\mathcal{O}}\left(1,2, B^{+}\right)$we first perform theorem 1 for the 1's and then for the 2's. This way we obtain terms of the form $[1 C 1]_{\varphi}[2 D 2]_{\varphi}$ with $A \backslash B^{+}=$ $(1,1,2,2, C, D)$ such that $D$ contains no 1 , and terms of the form $[1 C 1]_{\varphi}$ with $A \backslash B^{+}=(1,1,2, C)$. Using again the identities

$$
[1 C 1]_{\varphi}[2 D 2]_{\varphi}=\frac{[1 C 12 D 2]_{\varphi}}{\varphi_{12}}, \quad[1 C 1]_{\varphi}=\frac{[1 C 12]_{\varphi}}{\varphi_{12}}
$$

we obtain the desired statement. 


\section{One-particle expectation values}

In this section we calculate the one-particle mean value (4.3)-(4.4) using the results of the previous section for the degenerate form factors.

It follows from (2.12) that the asymptotic result is

$$
\langle\bar{\theta}|\mathcal{O}| \bar{\theta}\rangle=\frac{F_{2, c}^{\mathcal{O}}}{m L \cosh \bar{\theta}}+\langle\mathcal{O}\rangle .
$$

It is instructive to first obtain this asymptotic formula, neglecting all exponential corrections. This is presented in the following subsection. The exact result with all terms included is calculated in subsection 6.2.

\subsection{All orders in $1 / L$}

In the asymptotic approximation we only keep the contributions with $k=0$ from (4.3)-(4.4). These are the terms which do not contain the weight function $1 /\left(1+e^{\varepsilon(\theta)}\right) \sim e^{-m L}$ :

$$
\langle\bar{\theta}|\mathcal{O}| \bar{\theta}\rangle=\langle\mathcal{O}\rangle+\sum_{j=1}^{\infty} \frac{1}{j !} \frac{1}{n_{1}^{j}} F_{2 j, c}^{\mathcal{O}}(\bar{\theta}, \bar{\theta}, \ldots, \bar{\theta}) .
$$

In this approximation $n_{1}$ is

$$
n_{1}=m L \cosh \bar{\theta}+\varphi(0)+\mathcal{O}\left(e^{-m R}\right) .
$$

The relation (5.5) is used to sum up the second term in (6.2) as

$$
\begin{aligned}
& \sum_{j=1}^{\infty} \frac{1}{j !}\left(\frac{1}{m L \cosh \bar{\theta}+\varphi(0)}\right)^{j} F_{2 j, c}^{\mathcal{O}}(\bar{\theta}, \bar{\theta}, \ldots, \bar{\theta})= \\
& =\frac{F_{2, c}^{\mathcal{O}}}{m L \cosh \bar{\theta}+\varphi(0)} \sum_{j=1}^{\infty}\left(\frac{\varphi(0)}{m L \cosh \bar{\theta}+\varphi(0)}\right)^{j-1} \\
& =\frac{F_{2, c}^{\mathcal{O}}}{m L \cosh \bar{\theta}} \text {. }
\end{aligned}
$$

Putting together (6.2) and (6.3) we obtain indeed (6.1). It is interesting that a resummation of an infinite number of terms was required to obtain this simple formula.

\subsection{Exponential corrections}

Here we consider all terms in (4.3)-(4.4). The notations used in the following calculation are given at the beginning of subsection 5.1.

A given $\mathcal{L}_{j k}$ can be evaluated using the statement (5.4) for the degenerate form factors as

$$
\begin{aligned}
\mathcal{L}_{j k}= & \frac{1}{k !} \int \widetilde{d \theta_{2}} \ldots \widetilde{d \theta_{k+1}} n_{1}^{-j} \times \\
& \sum_{A_{k}=A^{-} \cup A^{+}} F_{2\left(1+\left|A^{+}\right|\right), c}^{\mathcal{O}}\left(\bar{\theta}+i \pi / 2, A^{+}\right) \sum_{s \in S_{11}\left(1(\times j), A^{-}\right)}[s]_{\varphi},
\end{aligned}
$$

where we assumed $j>1, A_{k}=\{2,3, \ldots, k+1\}$ and we identified $\theta_{1}=\bar{\theta}+i \pi / 2$.

Summing up all terms, grouping them according to the subset $A^{+}$and using the symmetry in the variables the mean value can be cast in the form

$$
\langle\bar{\theta}|\mathcal{O}| \bar{\theta}\rangle=\langle\mathcal{O}\rangle_{\varepsilon}+\frac{\sum_{l=0}^{\infty} \frac{1}{l !} \int \widetilde{d \theta_{2}} \ldots \widetilde{d \theta_{l+1}} F_{2(1+l), c}^{\mathcal{O}}\left(\bar{\theta}+i \pi / 2, \theta_{2}, \ldots, \theta_{l+1}\right)}{N_{1}}
$$


with

$$
\langle\mathcal{O}\rangle_{\varepsilon}=\sum_{n} \frac{1}{n !} \int \frac{d \theta_{1}}{2 \pi} \ldots \frac{d \theta_{n}}{2 \pi}\left(\prod_{j} \frac{1}{1+e^{\varepsilon\left(\theta_{j}\right)}}\right) F_{2 n, c}^{\mathcal{O}}\left(\theta_{1}, \ldots, \theta_{n}\right)
$$

and

$$
\frac{1}{N_{1}}=\frac{1}{n_{1}}+\sum_{j=2}^{\infty} \sum_{l=0}^{\infty} n_{1}^{-j} \frac{1}{l !} \int \widetilde{d \theta_{2}} \ldots \widetilde{d \theta_{l+1}} \sum_{s \in S_{11}(1(\times j), 2,3, \ldots, l+1)}[s]_{\varphi} .
$$

Here the first term $1 / n_{1}$ comes from those contributions where there is only a single insertion of $\bar{\theta}+i \pi / 2$. The infinite sum comes from the terms with multiple insertions.

On a sequence $s$ we define the function

$$
[s]_{1}=n_{1}^{-(m(1, s)-1)} \int \widetilde{d \theta_{2}} \ldots \widetilde{d \theta}_{l_{s}-m(1, s)+1}[s]_{\varphi} .
$$

Here $l_{s}$ denotes the total length of the sequence and $m(1, s)$ denotes the multiplicity of 1 in $s$. For example

$$
[11231]_{1}=\frac{1}{n_{1}^{2}} \int \widetilde{d \theta_{2}} \widetilde{d \theta_{3}} \varphi_{11} \varphi_{12} \varphi_{23} \varphi_{31}
$$

It is easy to see that under multiplication this function behaves as

$$
[1 A 1]_{1} \times[1 B 1]_{1}=[1 A 1 B 1]_{1},
$$

where $A$ and $B$ are arbitrary finite sequences.

Let $P_{1,1}$ be the set of finite sequences with the following properties: $s \in P_{1,1}$ if

1. Either $s=\left(1^{(\times j)}\right)$ with some $j \geq 2$ or $s$ is a permutation of the sequence

$$
\left(1^{(\times j)}, 2,3, \ldots, k\right)
$$

with some $j, k \geq 2$

2. $s$ starts and ends with 1 .

3. When numbers other than 1 are present, they appear in increasing order.

The first few examples are given below:

$$
\begin{aligned}
P_{1,1}= & \{(11),(111),(121),(1111),(1121),(1211),(1231), \\
& (11111),(12111),(11211),(11121),(11231),(12131),(12311),(12341), \ldots\} .
\end{aligned}
$$

With these notations (6.6) can be written as

$$
\frac{n_{1}}{N_{1}}=1+\sum_{s \in P_{1,1}}[s]_{1}
$$

Note that the property 3 in the definition of $P_{1,1}$ was needed to remove a factor of $1 / l$ ! from the expression (6.6).

Equation (6.9) presents an explicit representation of $N_{1}$, however it is possible to derive a simpler form. Let $T_{1,1}$ be the subset of $P_{1,1}$ containing only those sequences in which 1 appears only twice, at the beginning and at the end:

$$
T_{1,1}=\{(11),(121),(1231),(12341), \ldots\} .
$$

It can be considered the set of the "connected" components of $P_{1,1}$. 
Theorem 7. Inverting (6.6) gives

$$
\frac{N_{1}}{n_{1}}=1-\sum_{s \in T_{1,1}}[s]_{1}
$$

Proof. It needs to be proven that

$$
\sum_{s \in P_{1,1}}[s]_{1}-\sum_{s \in T_{1,1}}[s]_{1}=\left(\sum_{s \in T_{1,1}}[s]_{1}\right)\left(\sum_{s \in P_{1,1}}[s]_{1}\right) .
$$

In this form the l.h.s. consists of the sum of all allowed sequences in which the number 1 is present at least 3 times. Given an arbitrary such sequence $s$ it is possible to reconstruct it as $s=1 A 1 B 1$ such that $(1 A 1) \in T_{1,1}$ and $(1 B 1) \in P_{1,1}$. This provides a bijection between the elements on the l.h.s. and all possible products on the r.h.s. and using (6.8) we obtain a complete equality of the two sides.

Explicitly expanding $(6.10)$ we obtain

$$
\begin{aligned}
N_{1}= & n_{1}-\varphi(0)-\int \widetilde{d \theta_{2}} \varphi\left(\theta_{2}-\bar{\theta}-i \pi / 2\right)^{2} \\
& -\int \widetilde{d \theta_{2}} \widetilde{d \theta_{3}} \varphi\left(\theta_{2}-\bar{\theta}-i \pi / 2\right) \varphi\left(\theta_{2}-\theta_{3}\right) \varphi\left(\theta_{3}-\bar{\theta}-i \pi / 2\right)-\ldots
\end{aligned}
$$

Substituting the explicit representation (4.7) for $n_{1}$ results in

$$
\begin{aligned}
N_{1}= & m L\left(\cosh \bar{\theta}+\int \widetilde{d \theta} i \varphi(\theta-\bar{\theta}+i \pi / 2) \sinh \theta\right. \\
& \left.+\sum_{n=2}^{\infty} \int \widetilde{d \theta_{1}} \ldots \widetilde{d \theta_{n}} i \varphi\left(\theta_{1}-\bar{\theta}+i \pi / 2\right) \varphi\left(\theta_{1}-\theta_{2}\right) \ldots \varphi\left(\theta_{n-1}-\theta_{n}\right) \sinh \theta_{n}\right) .
\end{aligned}
$$

This is our final formula for the normalization factor in (6.4).

\subsection{Interpretation of the result}

Consider the function $\bar{Q}(\bar{\theta})$ defined in (4.2). Taking the total derivative with respect to $\bar{\theta}$

$$
\frac{d \bar{Q}}{d \bar{\theta}}=m L \cosh \bar{\theta}+\int \frac{d \theta^{\prime}}{2 \pi} i \varphi\left(\theta^{\prime}-\bar{\theta}+i \pi / 2\right) \frac{1}{1+e^{\varepsilon\left(\theta^{\prime}\right)}}\left(\frac{\partial \varepsilon}{\partial \theta}+\frac{\partial \varepsilon}{\partial \bar{\theta}}\right) .
$$

Here it is understood that the pseudoenergy function $\varepsilon(\theta)$ also depends on $\bar{\theta}$ through the source term in (3.1).

Differentiating (3.4)

$$
(1-\hat{K})\left(\frac{\partial \varepsilon}{\partial \theta}+\frac{\partial \varepsilon}{\partial \bar{\theta}}\right)=m L \sinh \theta
$$

with $K\left(\theta, \theta^{\prime}\right)=\varphi\left(\theta-\theta^{\prime}\right)$. Inverting the integral equation and substituting into (6.12)

$$
\begin{aligned}
\frac{\partial \bar{Q}}{\partial \bar{\theta}}= & m L\left(\cosh \bar{\theta}+\int \widetilde{d \theta} i \varphi(\theta-\bar{\theta}+i \pi / 2) \sinh \theta\right. \\
& \left.+\sum_{n=2}^{\infty} \int \widetilde{d \theta_{1}} \ldots \widetilde{d \theta_{n}} i \varphi\left(\theta_{1}-\bar{\theta}+i \pi / 2\right) \varphi\left(\theta_{1}-\theta_{2}\right) \ldots \varphi\left(\theta_{n-1}-\theta_{n}\right) \sinh \theta_{n}\right)= \\
= & N_{1} .
\end{aligned}
$$


In other words, the normalization factor obtained in the previous subsection coincides with the exact total derivative of the quantization condition for the rapidity $\bar{\theta}$. This is analogous to the asymptotic result (2.12), which involves the derivatives of the (asymptotic) Bethe equations.

To conclude this section we present the one-particle result in a compact formula:

$$
\begin{aligned}
& \langle\bar{\theta}|\mathcal{O}| \bar{\theta}\rangle_{L}= \\
& \sum_{n=0}^{\infty} \frac{1}{n !} \int \frac{d \theta_{1}}{2 \pi} \ldots \frac{d \theta_{n}}{2 \pi}\left(\prod_{j} \frac{1}{1+e^{\varepsilon\left(\theta_{j}\right)}}\right) F_{2 n, c}^{\mathcal{O}}\left(\theta_{1}, \ldots, \theta_{n}\right) \\
& +\left(\frac{\partial \bar{Q}}{\partial \bar{\theta}}\right)^{-1} \sum_{n=0}^{\infty} \frac{1}{n !} \int \frac{d \theta_{1}}{2 \pi} \ldots \frac{d \theta_{n}}{2 \pi}\left(\prod_{j} \frac{1}{1+e^{\varepsilon\left(\theta_{j}\right)}}\right) F_{2(n+1), c}^{\mathcal{O}}\left(\bar{\theta}+i \pi / 2, \theta_{1}, \ldots, \theta_{n}\right) .
\end{aligned}
$$

The infinite series in the second line above is a result of the terms $\mathcal{L}_{j k}$ with $j=0$ and it has exactly the same form as the original LeClair-Mussardo series; the only difference is that the excited state pseudoenergy function is used in the weight functions. The third line is an

analogous series, with the two differences being the presence of the rapidity $\bar{\theta}+i \pi / 2$ and the normalization factor which is of order $1 / L$.

\section{Two-particle expectation values}

Here we perform the partial summation of the expansion (4.12)-(4.13) for the two-particle mean-value. The asymptotic result following from (2.12) is

$$
\begin{aligned}
& \left\langle\bar{\theta}_{1}, \bar{\theta}_{2}|\mathcal{O}| \bar{\theta}_{1}, \bar{\theta}_{2}\right\rangle_{L}=\langle\mathcal{O}\rangle+ \\
& \quad+\frac{F_{4 c}^{\mathcal{O}}\left(\bar{\theta}_{1}, \bar{\theta}_{2}\right)+F_{4 c}^{\mathcal{O}}\left(\bar{\theta}_{1}\right)\left(m L \cosh \bar{\theta}_{2}+\varphi_{12}\right)+F_{4 c}^{\mathcal{O}}\left(\bar{\theta}_{2}\right)\left(m L \cosh \bar{\theta}_{1}+\varphi_{12}\right)}{\rho_{2}\left(\bar{\theta}_{1}, \bar{\theta}_{2}\right)},
\end{aligned}
$$

with

$$
\rho_{2}\left(\bar{\theta}_{1}, \bar{\theta}_{2}\right)=m^{2} L^{2} \cosh \bar{\theta}_{1} \cosh \bar{\theta}_{2}+m L\left(\cosh \bar{\theta}_{1}+\cosh \bar{\theta}_{2}\right) \varphi_{12} .
$$

The summation procedure to obtain this asymptotic result is already involved and is presented in the following subsection. The evaluation of the exact result (4.12)-(4.13) with all exponential corrections included is presented in subsection 7.2 .

\subsection{All orders in $1 / L$}

Dropping the exponential corrections amounts to summing up the terms with $l=0$ in (4.12). This leads to

$$
\begin{aligned}
\left\langle\bar{\theta}_{1}, \bar{\theta}_{2}|\mathcal{O}| \bar{\theta}_{1}, \bar{\theta}_{2}\right\rangle= & \sum_{j, k=1}^{\infty} \mathcal{L}_{j k 0}= \\
& =\langle\mathcal{O}\rangle+\sum_{m=1}^{\infty} \frac{1}{m !} \sum_{i_{1} \ldots i_{m}=1}^{2} \frac{F_{2 m, c}^{\mathcal{O}}\left(\bar{\theta}_{i_{1}}, \bar{\theta}_{i_{2}}, \ldots, \bar{\theta}_{i_{m}}\right)}{n_{i_{1}} n_{i_{2}} \ldots n_{i_{m}}}
\end{aligned}
$$

where the normalization factors in this approximation are

$$
\frac{1}{n_{j}} \equiv \operatorname{Res}_{\theta=\bar{\theta}_{j}} \frac{1}{1+e^{\varepsilon(\theta)}}=\frac{1}{m L \cosh \bar{\theta}_{j}+\varphi(0)+\varphi\left(\bar{\theta}_{1}-\bar{\theta}_{2}\right)}+\mathcal{O}\left(e^{-m R}\right), \quad j=1,2 .
$$

Let us denote by $\mathcal{L}_{j}$ the sum of the terms which include $j$ rapidities in total:

$$
\mathcal{L}_{j}=\sum_{k+l=j} \mathcal{L}_{k l 0}
$$


The first few cases can be evaluated using theorem 5 explicitly as

$$
\begin{aligned}
\mathcal{L}_{1}= & F_{2 c}^{\mathcal{O}}\left(\frac{1}{n_{1}}+\frac{1}{n_{2}}\right) \\
\mathcal{L}_{2}= & F_{2 c}^{\mathcal{O}}\left(\frac{\varphi_{0}}{n_{1}^{2}}+\frac{\varphi_{0}}{n_{2}^{2}}\right)+F_{4 c}^{\mathcal{O}}(1,2) \frac{1}{n_{1} n_{2}} \\
\mathcal{L}_{3}= & F_{2 c}^{\mathcal{O}}\left(\frac{\varphi_{0}^{2}}{n_{1}^{3}}+\frac{\varphi_{0}^{2}}{n_{2}^{3}}+\frac{\varphi_{12}^{2}}{n_{1}^{2} n_{2}}+\frac{\varphi_{12}^{2}}{n_{2}^{2} n_{1}}\right) \\
& +F_{4 c}^{\mathcal{O}}(1,2)\left(\frac{\varphi_{0}}{n_{1}^{2} n_{2}}+\frac{\varphi_{0}}{n_{2}^{2} n_{1}}\right) \\
\mathcal{L}_{4}= & F_{2 c}^{\mathcal{O}}\left(\frac{\varphi_{0}^{3}}{n_{1}^{4}}+\frac{\varphi_{0}^{3}}{n_{2}^{4}}+\frac{2 \varphi_{12}^{2} \varphi_{0}}{n_{1}^{3} n_{2}}+\frac{2 \varphi_{12}^{2} \varphi_{0}}{n_{2}^{3} n_{1}}+\frac{2 \varphi_{12}^{2} \varphi_{0}}{n_{2}^{2} n_{1}^{2}}\right) \\
& +F_{4 c}^{\mathcal{O}}(1,2)\left(\frac{\varphi_{0}^{2}}{n_{1}^{3} n_{2}}+\frac{\varphi_{0}^{2}}{n_{2}^{3} n_{1}}+\frac{\varphi_{0}^{2}+\varphi_{12}^{2}}{n_{1}^{2} n_{2}^{2}}\right) .
\end{aligned}
$$

All terms in this series can be obtained by an auxiliary problem. Let $A_{i}, i=0 \ldots \infty$ a series of $2 \times 2$ matrices satisfying the recursion relation

$$
A_{i+1}=\left(\begin{array}{cc}
\frac{1}{n_{1}} & 0 \\
0 & \frac{1}{n_{2}}
\end{array}\right)+\left(\begin{array}{ll}
\frac{\varphi_{0}}{n_{1}} & \frac{\varphi_{12}}{n_{1}} \\
\frac{\varphi_{12}}{n_{2}} & \frac{\varphi_{0}}{n_{2}}
\end{array}\right) A_{i}, \quad A_{0}=0 .
$$

Theorem 8. The summation of the series $\mathcal{L}_{j}$ to a given order $k$ is given by

$$
\sum_{j=0}^{k} \mathcal{L}_{j}=\operatorname{Tr}\left[\left(\begin{array}{cc}
F_{2 c}^{\mathcal{O}} & \frac{F_{4 c}^{\mathcal{O}}(1,2)}{2 \varphi_{12}} \\
\frac{F_{4 c}^{\mathcal{O}}(1,2)}{2 \varphi_{12}} & F_{2 c}^{\mathcal{O}}
\end{array}\right) A_{k}\right]
$$

Proof. The matrix multiplication in (7.3) generates the sum over all possible paths consisting of the numbers 1 and 2. It also generates the proper normalization factors which appear in (7.2). The statement of the theorem then follows from Theorem (5).

Given that $L$ is large enough the expressions in (7.4) are convergent and

$$
\sum_{j=0}^{\infty} \mathcal{L}_{j}=\operatorname{Tr}\left[\left(\begin{array}{cc}
F_{2 c}^{\mathcal{O}} & \frac{F_{4 c}^{\mathcal{O}}(1,2)}{2 \varphi_{12}} \\
\frac{F_{4 c}^{\mathcal{O}}(1,2)}{2 \varphi_{12}} & F_{2 c}^{\mathcal{O}}
\end{array}\right) A_{\infty}\right], \quad \text { with } \quad A_{\infty}=\lim _{j \rightarrow \infty} A_{j}
$$

The limiting matrix $A_{\infty}$ can be obtained from (7.3) as

$$
A_{\infty}=\left(I-\left(\begin{array}{ll}
\frac{\varphi_{0}}{n_{1}} & \frac{\varphi_{12}}{n_{1}} \\
\frac{\varphi_{12}}{n_{2}} & \frac{\varphi_{0}}{n_{2}}
\end{array}\right)\right)^{-1}\left(\begin{array}{cc}
\frac{1}{n_{1}} & 0 \\
0 & \frac{1}{n_{2}}
\end{array}\right)=\frac{1}{\rho_{2}}\left(\begin{array}{cc}
m L \cosh \bar{\theta}_{2}+\varphi_{12} & \varphi_{12} \\
\varphi_{12} & m L \cosh \bar{\theta}_{1}+\varphi_{12}
\end{array}\right) .
$$

Putting together (7.5) and (7.6) leads to the desired statement (7.1).

\subsection{Exponential corrections}

Here we use theorem 6 to evaluate the expansion (4.12)-(4.13). The terms will be grouped according to the indices $j$ and $k$.

The terms with no insertions of the external rapidities are $\mathcal{L}_{0,0, l}$ with $l=0 \ldots \infty$ :

$$
\sum_{l=0}^{\infty} \mathcal{L}_{0,0, l} \equiv\langle\mathcal{O}\rangle_{\varepsilon}=\sum_{n} \frac{1}{n !} \int \frac{d \theta_{1}}{2 \pi} \ldots \frac{d \theta_{n}}{2 \pi}\left(\prod_{j} \frac{1}{1+e^{\varepsilon\left(\theta_{j}\right)}}\right) F_{2 n, c}^{\mathcal{O}}\left(\theta_{1}, \ldots, \theta_{n}\right) .
$$


The terms $\mathcal{L}_{1,0, l}, \mathcal{L}_{0,1, l}$ and $\mathcal{L}_{1,1, l}$ are simply

$$
\begin{aligned}
& \mathcal{L}_{1,0, l}=\frac{1}{n_{1}} \frac{1}{l !} \int \widetilde{d \theta_{3}} \ldots \widetilde{d \theta_{l+2}} F_{2(1+l), c}^{\mathcal{O}}\left(\bar{\theta}_{1}+i \pi / 2, \theta_{3}, \ldots, \theta_{l+2}\right) \\
& \mathcal{L}_{0,1, l}=\frac{1}{n_{2}} \frac{1}{l !} \int \widetilde{d \theta_{3}} \ldots \widetilde{d \theta_{l+2}} F_{2(1+l), c}^{\mathcal{O}}\left(\bar{\theta}_{2}+i \pi / 2, \theta_{3}, \ldots, \theta_{l+2}\right) \\
& \mathcal{L}_{1,1, l}=\frac{1}{n_{1} n_{2}} \frac{1}{l !} \int \widetilde{d \theta_{3}} \ldots \widetilde{d \theta_{l+2}} F_{2(2+l), c}^{\mathcal{O}}\left(\bar{\theta}_{1}+i \pi / 2, \bar{\theta}_{2}+i \pi / 2, \theta_{3}, \ldots, \theta_{l+2}\right) .
\end{aligned}
$$

The terms $\mathcal{L}_{j, 0, l}$ and $\mathcal{L}_{0, j, l}$ with $j \geq 2$, ie. those with multiple insertions of either one of $\bar{\theta}_{1,2}+i \pi / 2$ can be evaluated using Theorem 国:

$$
\begin{aligned}
\mathcal{L}_{j, 0, l}= & \frac{1}{k !} \int \widetilde{d \theta_{3}} \ldots \widetilde{d \theta_{k+2}} n_{1}^{-j} \times \\
& \sum_{A_{k}=A^{-} \cup A^{+}} F_{2\left(1+\left|A^{+}\right|\right), c}^{\mathcal{O}\left(\bar{\theta}_{1}+i \pi / 2, A^{+}\right)} \sum_{s \in S_{11}\left(1(\times j), A^{-}\right)}[s]_{\varphi},
\end{aligned}
$$

where we assumed $j>1, A_{k}=\{3, \ldots, k+2\}$ and in the notation for the sequences we identified $\theta_{1} \equiv \bar{\theta}_{1}+i \pi / 2$.

Similarly

$$
\begin{aligned}
\mathcal{L}_{0, j, l}= & \frac{1}{k !} \int \widetilde{d \theta_{3}} \ldots \widetilde{d \theta_{k+2}} n_{2}^{-j} \times \\
& \sum_{A_{k}=A^{-} \cup A^{+}} F_{2\left(1+\left|A^{+}\right|\right), c}^{\mathcal{O}}\left(\bar{\theta}_{2}+i \pi / 2, A^{+}\right) \sum_{s \in S_{22}\left(2^{(\times j)}, A^{-}\right)}[s]_{\varphi},
\end{aligned}
$$

with $\theta_{2} \equiv \bar{\theta}_{2}+i \pi / 2$.

The terms $\mathcal{L}_{j, 1, l}$ can also be evaluated using Theorem 4 :

$$
\begin{aligned}
\mathcal{L}_{j, 1, l}=\frac{1}{k !} \int \widetilde{d \theta_{3}} & \ldots \widetilde{d \theta_{k+2}} \frac{1}{n_{1}^{j} n_{2}} \times \\
\sum_{A_{k}=A^{-} \cup A^{+}}( & F_{2\left(2+\left|A^{+}\right|\right), c}^{\mathcal{O}}\left(\bar{\theta}_{1}+i \pi / 2, \bar{\theta}_{2}+i \pi / 2, A^{+}\right) \sum_{s \in S_{11}\left(1^{(\times j)}, A^{-}\right)}[s]_{\varphi} \\
& \left.+F_{2\left(1+\left|A^{+}\right|\right), c}^{\mathcal{O}}\left(\bar{\theta}_{1}+i \pi / 2, A^{+}\right) \sum_{s \in S_{11}\left(1^{(\times j)}, 2, A^{-}\right)}[s]_{\varphi}\right)
\end{aligned}
$$

where again $A_{k}=\{3, \ldots, k+2\}$. An analogous expression holds for the terms $\mathcal{L}_{1, j, l}$ with the role of the numbers 1 and 2 interchanged.

Finally Theorem 6 yields the terms with multiple insertions of both external rapidities:

$$
\begin{aligned}
\mathcal{L}_{j, k, l}=\frac{1}{l !} \int \widetilde{d \theta_{3}} & \ldots \widetilde{d \theta_{l+2}} \frac{1}{n_{1}^{j} n_{2}^{k}} \times \\
\sum_{A_{l}=A^{-} \cup A^{+}}( & F_{2\left(1+\left|A^{+}\right|\right), c}^{\mathcal{O}}\left(\bar{\theta}_{1}+i \pi / 2, A^{+}\right) \sum_{s \in S_{11}\left(1(\times j), 2^{(\times k)}, A^{-}\right)}[s]_{\varphi} \\
& +F_{2\left(1+\left|A^{+}\right|\right), c}^{\mathcal{O}}\left(\bar{\theta}_{2}+i \pi / 2, A^{+}\right) \sum_{s \in S_{22}\left(1(\times j), 2^{(\times k)}, A^{-}\right)}[s]_{\varphi} \\
& \left.+\frac{1}{\varphi_{12}} F_{2\left(2+\left|A^{+}\right|\right), c}^{\mathcal{O}}\left(\bar{\theta}_{1}+i \pi / 2, \bar{\theta}_{2}+i \pi / 2, A^{+}\right) \sum_{s \in \tilde{S}_{12}\left(1(\times j), 2(\times k), A^{-}\right)}[s]_{\varphi}\right),
\end{aligned}
$$

with $A_{l}=\{3, \ldots, l+2\}$. 
Adding all the terms above and grouping them according to the form factor content we obtain the expansion

$$
\begin{aligned}
\left\langle\bar{\theta}_{1}, \bar{\theta}_{2}|\mathcal{O}| \bar{\theta}_{1}, \bar{\theta}_{2}\right\rangle_{L}=\langle\mathcal{O}\rangle_{\varepsilon}+ & \\
+ & \frac{\sum_{l=0}^{\infty} \frac{1}{k !} \int \widetilde{d \theta_{3}} \ldots \widetilde{d \theta_{k+2}} F_{2(1+k), c}^{\mathcal{O}}\left(\bar{\theta}_{1}+i \pi / 2, \theta_{3}, \ldots, \theta_{k+2}\right)}{N_{1}} \\
& +\frac{\sum_{k=0}^{\infty} \frac{1}{k !} \int \widetilde{d \theta_{3}} \ldots \widetilde{d \theta_{k+2}} F_{2(1+k), c}^{\mathcal{O}}\left(\bar{\theta}_{2}+i \pi / 2, \theta_{3}, \ldots, \theta_{k+2}\right)}{N_{2}} \\
& +\frac{\sum_{k=0}^{\infty} \frac{1}{k !} \int \widetilde{d \theta_{3}} \ldots \widetilde{d \theta_{k+2}} F_{2(2+k), c}^{\mathcal{O}}\left(\bar{\theta}_{1}+i \pi / 2, \bar{\theta}_{2}+i \pi / 2, \theta_{3}, \ldots, \theta_{k+2}\right)}{N_{12}},
\end{aligned}
$$

where the normalization factors are

$$
\begin{aligned}
& \frac{1}{N_{1}}=\frac{1}{n_{1}}+\sum_{j=2}^{\infty} \sum_{l=0}^{\infty} \frac{1}{n_{1}^{j}} \frac{1}{l !} \int \widetilde{d \theta_{3}} \ldots \widetilde{d \theta_{l+2}} \sum_{s \in S_{11}(1(\times j), 3, \ldots, l+2)}[s]_{\varphi} \\
& +\sum_{j=2}^{\infty} \sum_{k=1}^{\infty} \sum_{l=0}^{\infty} \frac{1}{n_{1}^{j} n_{2}^{k}} \frac{1}{l !} \int \widetilde{d \theta_{3}} \ldots \widetilde{d \theta_{l+2}} \sum_{s \in S_{11}\left(1^{(\times j)}, 2(\times k), 3, \ldots, l+2\right)}[s]_{\varphi}, \\
& \frac{1}{N_{2}}=\frac{1}{n_{2}}+\sum_{j=2}^{\infty} \sum_{l=0}^{\infty} \frac{1}{n_{2}^{j}} \frac{1}{l !} \int \widetilde{d \theta_{3}} \ldots \widetilde{d \theta_{l+2}} \sum_{s \in S_{22}(2(\times j), 3, \ldots, l+2)}[s]_{\varphi} \\
& +\sum_{j=2}^{\infty} \sum_{k=1}^{\infty} \sum_{l=0}^{\infty} \frac{1}{n_{2}^{j} n_{1}^{k}} \frac{1}{l !} \int \widetilde{d \theta_{3}} \ldots \widetilde{d \theta_{l+2}} \sum_{s \in S_{22}\left(1^{(\times k)}, 2^{(\times j)}, 3, \ldots, l+2\right)}[s]_{\varphi}
\end{aligned}
$$

and finally

$$
\begin{aligned}
\frac{1}{N_{12}}= & \frac{1}{n_{1} n_{2}}+\sum_{j=2}^{\infty} \sum_{l=0}^{\infty} \frac{1}{n_{1}^{j} n_{2}} \frac{1}{l !} \int \widetilde{d \theta_{3}} \ldots \widetilde{d \theta_{l+2}} \sum_{s \in S_{11}(1(\times j), 3, \ldots, l+2)}[s]_{\varphi} \\
& +\sum_{j=2}^{\infty} \sum_{l=0}^{\infty} \frac{1}{n_{1} n_{2}^{j}} \frac{1}{l !} \int \widetilde{d \theta_{3}} \ldots \widetilde{d \theta_{l+2}} \sum_{s \in S_{22}\left(2^{(\times j)}, 3, \ldots, l+2\right)}[s]_{\varphi} \\
& +\frac{1}{\varphi_{12}} \sum_{j=2}^{\infty} \sum_{k=2}^{\infty} \sum_{l=0}^{\infty} \frac{1}{n_{1}^{j} n_{2}^{k}} \frac{1}{l !} \int \widetilde{d \theta_{3}} \ldots \widetilde{d \theta_{l+2}} \sum_{s \in \tilde{S}_{12}(1(\times j), 2(\times k), 3, \ldots, l+2)}[s]_{\varphi} .
\end{aligned}
$$

The double and triple sums in the previous three formulas can be simplified dramatically. However, first we have to introduce new notations motivated by the expressions above.

Let $R_{a, b}, a, b=1,2$ be four sets of finite sequences with the following properties: $s \in R_{a, b}$ if

1. Either $s=\left(1^{(\times j)}\right)$ or $s=\left(2^{(\times j)}\right)$ with some $j \geq 2$, or $s$ is a permutation of the sequences

$$
\left(1^{(\times j)}, 2^{(\times k)}\right)
$$

with some $j \geq 1, k \geq 1$, or

$$
\left(1^{(\times j)}, 2^{(\times k)}, 3, \ldots, l\right)
$$

with some $j+k \geq 2$ and $l \geq 3$.

2. $s$ starts with $a$ and ends with $b$.

3. When numbers other than 1 and 2 are present, they appear in increasing order. 
The difference between the sets $R_{a, b}$ and the set $P_{1,1}$ introduced in section 6.2 is that in the sequences in $R_{a, b}$ the number 2 can appear multiple times at arbitrary positions and only the ordering of the numbers greater than 2 is constrained.

It is useful to introduce a shorthand for the integrals entering (7.9)-(7.11). We define

$$
[s]_{12}=n_{1}^{-m(1, s)} n_{2}^{-m(2, s)} n_{s\left(l_{s}\right)} \int \widetilde{d \theta}_{3} \ldots \widetilde{d \theta}_{l_{s}-l(1, s)-l(2, s)}[s]_{\varphi},
$$

where $m(1, s)$ and $m(2, s)$ denote the multiplicity of the numbers 1 and 2 in $s$, respectively and $l_{s}$ is simply the length of $s$ (therefore $s\left(l_{s}\right)$ denotes the last element of the sequence). For example

$$
[21321]_{12}=\frac{1}{n_{1} n_{2}^{2}} \varphi_{12}^{2} \int \widetilde{d \theta_{3}} \varphi_{23} \varphi_{13}
$$

This function satisfies

$$
[a A b]_{12} \times[b B c]_{12}=[a A b B c]_{12}
$$

for every $a, b, c=1,2$ and $A, B$ being arbitrary finite sequences.

With these notations the first two normalization factors in (7.8) can be expressed as

$$
\begin{aligned}
& \frac{1}{N_{1}}=\frac{1}{n_{1}}+\frac{1}{n_{1}} \sum_{s \in R_{1,1}}[s]_{12} \\
& \frac{1}{N_{2}}=\frac{1}{n_{2}}+\frac{1}{n_{2}} \sum_{s \in R_{2,2}}[s]_{12} .
\end{aligned}
$$

It is also possible to obtain a compact formula for $N_{12}$ expressed as a simple sum. We define $\tilde{R}_{1,2}$ to be the subset of $R_{1,2}$ which includes sequences where the first number after the rightmost 1 is 2 . The first few sequences are

$$
\tilde{R}_{1,2}=\{(12),(112),(122),(1112),(1122),(1222),(1232),(1312), \ldots\} .
$$

\section{Theorem 9.}

$$
\frac{1}{N_{12}}=\frac{1}{\varphi_{12}} \frac{1}{n_{2}} \sum_{s \in \tilde{R}_{1,2}}[s]_{12} .
$$

Proof. The idea of the proof is to show that each term in (7.11) corresponds to one of the elements of $\tilde{R}_{1,2}$ and that (7.16) provides the correct normalization factors.

The first term in (7.11) corresponds to the sequence $(12) \in \tilde{R}_{1,2}$. We have by definition

$$
\frac{1}{n_{1} n_{2}}=\frac{1}{\varphi_{12}} \frac{[12]_{\varphi}}{n_{1} n_{2}}
$$

Concerning the second term in the r.h.s. of (7.11) note that if $s \in S_{11}\left(1^{(\times j)}, 3, \ldots, l+2\right)$ then the sequence $(s 2)$ satisfies

$$
(s 2) \in \tilde{S}_{12}\left(1^{(\times j)}, 2,3, \ldots, l+2\right), \quad[s 2]_{\varphi}=\varphi_{12}[s]_{\varphi} .
$$

Similarly, if $s \in S_{22}\left(2^{(\times j)}, 3, \ldots, l+2\right)$ then the sequence $(1 s)$ satisfies

$$
(1 s) \in \tilde{S}_{12}\left(1,2^{(\times j)}, 3, \ldots, l+2\right), \quad[1 s]_{\varphi}=\varphi_{12}[s]_{\varphi} .
$$

These two cases give the sequences of $\tilde{R}_{1,2}$ in which the number 2 (or 1 ) appears only once, respectively.

Finally the the last line of (7.11) yields all the remaining sequences, where both 1 and 2 appear at least twice.

Using the definition (7.12) we obtain the statement of the theorem. 
We introduce the four sets of sequences $T_{a, b}$ with $a, b=1,2$ :

$$
T_{a, b} \equiv\{(a b),(a 3 b),(a 34 b),(a 345 b), \ldots\} .
$$

Consider the auxiliary problem

$$
A_{i+1}=\left(\begin{array}{cc}
\frac{1}{n_{1}} & 0 \\
0 & \frac{1}{n_{2}}
\end{array}\right)+M \times A_{i} \quad A_{0}=0
$$

with

$$
M=\left(\begin{array}{ll}
\sum_{s \in T_{1,1}}[s]_{12} & \sum_{s \in T_{1,2}}[s]_{12} \\
\sum_{s \in T_{2,1}}[s]_{12} & \sum_{s \in T_{2,2}}[s]_{12}
\end{array}\right) .
$$

This recursion relation generates all sequences of integers which are elements of the sets $R_{a, b}$.

Theorem 10. The limiting matrix $A_{\infty}$ has the following diagonal elements:

$$
\left(A_{\infty}\right)_{1,1}=\frac{1}{N_{1}} \quad\left(A_{\infty}\right)_{2,2}=\frac{1}{N_{2}} .
$$

Proof. The statement of the theorem follows from the property (7.13).

Theorem 11. The off-diagonal element $\left(A_{\infty}\right)_{1,2}$ is

$$
\left(A_{\infty}\right)_{1,2}=\frac{n_{1}}{n_{2}} \frac{1}{\varphi_{12}} \sum_{s \in T_{1,2}}[s]_{12} \sum_{s \in \tilde{R}_{1,2}}[s]_{12} .
$$

Proof. The iterative procedure gives

$$
\left(A_{\infty}\right)_{1,2}=\frac{1}{n_{2}} \sum_{s \in R_{1,2}}[s]_{12} .
$$

A given sequence $s \in R_{1,2}$ can be written as

$$
s=(A 1 C 2 B),
$$

such that if $A$ is not empty then it starts with 1 , if $B$ is not empty then it ends with 2 , and neither $B$ nor $C$ contain any 1's. Using the identity

$$
[A 1 C 2 B]_{12}=\frac{n_{1}}{\varphi_{12}}[A 12 B]_{12}[1 C 2]_{12}
$$

we obtain an exact bijection between the two sides of (7.20).

We introduce the quantities

$$
\mathcal{N}_{\varphi} \equiv n_{1} \sum_{s \in T_{1,2}}[s]_{12}
$$

and

$$
\mathcal{N}_{j} \equiv n_{j}\left(1-M_{j, j}\right)-\mathcal{N}_{\varphi} \quad j=1,2 .
$$

The explicit form of $\mathcal{N}_{\varphi}$ is

$$
\begin{aligned}
\mathcal{N}_{\varphi} & =\varphi\left(\bar{\theta}_{1}-\bar{\theta}_{2}\right)+\int \widetilde{d \theta} \varphi\left(\theta-\bar{\theta}_{1}-i \pi / 2\right) \varphi\left(\theta-\bar{\theta}_{2}-i \pi / 2\right)+ \\
& +\sum_{n=2}^{\infty} \int \widetilde{d \theta_{1}} \ldots \widetilde{d \theta_{n}} \varphi\left(\theta_{1}-\bar{\theta}_{1}-i \pi / 2\right) \varphi 12 \ldots \varphi_{n-1, n} \varphi\left(\theta_{n}-\bar{\theta}_{2}-i \pi / 2\right)
\end{aligned}
$$


On the other hand, using the definitions (7.21) and (7.18) and the explicit form of $n_{1,2}$ given by (4.11) we obtain the integral series

$$
\begin{aligned}
& \mathcal{N}_{j}=m \cosh \bar{\theta}_{j}+\int \widetilde{d \theta} i \varphi\left(\theta-\bar{\theta}_{j}+i \pi / 2\right) m \sinh \theta+ \\
&+\sum_{n=2}^{\infty} \int \widetilde{d \theta_{1}} \ldots \widetilde{d \theta_{n}} i \varphi\left(\theta_{1}-\bar{\theta}_{j}+i \pi / 2\right) \varphi_{12} \ldots \varphi_{n-1, n} m \sinh \theta_{n} \\
& \quad \text { with } j=1,2 .
\end{aligned}
$$

With these notations the limiting matrix $A_{\infty}$ can be obtained as

$$
A_{\infty}=(I-M)^{-1}\left(\begin{array}{cc}
\frac{1}{n_{1}} & 0 \\
0 & \frac{1}{n_{2}}
\end{array}\right)=\frac{1}{\mathcal{N}_{1} \mathcal{N}_{2}+\left(\mathcal{N}_{1}+\mathcal{N}_{2}\right) \mathcal{N}_{\varphi}}\left(\begin{array}{cc}
\mathcal{N}_{2}+\mathcal{N}_{\varphi} & \mathcal{N}_{\varphi} \\
\mathcal{N}_{\varphi} & \mathcal{N}_{1}+\mathcal{N}_{\varphi}
\end{array}\right)
$$

Putting together (7.8), (7.19), (7.16) and (7.20) we obtain our final formula

$$
\begin{aligned}
\left\langle\bar{\theta}_{1}, \bar{\theta}_{2}|\mathcal{O}| \bar{\theta}_{1}, \bar{\theta}_{2}\right\rangle_{L}=\langle\mathcal{O}\rangle_{\varepsilon}+\frac{1}{\mathcal{N}_{1} \mathcal{N}_{2}+\left(\mathcal{N}_{1}+\mathcal{N}_{2}\right) \mathcal{N}_{\varphi}}\{ \\
+\left(\mathcal{N}_{2}+\mathcal{N}_{\varphi}\right) \sum_{k=0}^{\infty} \frac{1}{k !} \int \widetilde{d \theta_{1}} \ldots \widetilde{d \theta_{k}} F_{2(1+k), c}^{\mathcal{O}}\left(\bar{\theta}_{1}+i \pi / 2, \theta_{1}, \ldots, \theta_{k}\right) \\
+\left(\mathcal{N}_{1}+\mathcal{N}_{\varphi}\right) \sum_{k=0}^{\infty} \frac{1}{k !} \int \widetilde{d \theta_{1}} \ldots \widetilde{d \theta_{k}} F_{2(1+k), c}^{\mathcal{O}}\left(\bar{\theta}_{2}+i \pi / 2, \theta_{1}, \ldots, \theta_{k}\right) \\
\left.+\sum_{k=0}^{\infty} \frac{1}{k !} \int \widetilde{d \theta_{1}} \ldots \widetilde{d \theta_{k}} F_{2(2+k), c}^{\mathcal{O}}\left(\bar{\theta}_{1}+i \pi / 2, \bar{\theta}_{2}+i \pi / 2, \theta_{1}, \ldots, \theta_{k}\right)\right\} .
\end{aligned}
$$

\subsection{Interpretation of the result}

The quantities $\mathcal{N}_{1}, \mathcal{N}_{2}$ and $\mathcal{N}_{\varphi}$ defined above appear to be dressed versions of the elements of the Jacobian of the two-particle Bethe equations. In the following we show that they are indeed the appropriate total derivatives of the two-particle quantization conditions as defined by the excited state TBA.

We define

$$
\bar{Q}_{j}=-i \varepsilon\left(\bar{\theta}_{j}+i \pi / 2\right), \quad j=1,2,
$$

and

$$
\mathcal{K}_{j k} \equiv \frac{\partial \bar{Q}_{j}}{\partial \bar{\theta}_{k}}
$$

Here it is understood that a total derivative has to be taken, including the dependence of the pseudoenergy function on the roots $\bar{\theta}_{1,2}$.

The diagonal elements of the Jacobian are given by

$$
\mathcal{K}_{j j}=m L \cosh \bar{\theta}_{j}+\varphi\left(\bar{\theta}_{1}-\bar{\theta}_{2}\right)-\int \frac{d \theta^{\prime}}{2 \pi} i \varphi\left(\theta^{\prime}-\bar{\theta}_{j}-i \pi / 2\right) \frac{1}{1+e^{\varepsilon\left(\theta^{\prime}\right)}}\left(\frac{\partial \varepsilon}{\partial \theta}+\frac{\partial \varepsilon}{\partial \bar{\theta}_{j}}\right), \quad j=1,2 .
$$

For the off-diagonal elements

$$
\mathcal{K}_{j k}=-\varphi\left(\bar{\theta}_{j}-\bar{\theta}_{k}\right)-\int \frac{d \theta^{\prime}}{2 \pi} i \varphi\left(\theta^{\prime}-\bar{\theta}_{j}-i \pi / 2\right) \frac{1}{1+e^{\varepsilon\left(\theta^{\prime}\right)}} \frac{\partial \varepsilon}{\partial \bar{\theta}_{k}}, \quad j \neq k .
$$

It follows from (4.8) that the derivatives of the pseudoenergy satisfy

$$
\begin{aligned}
& (1-\hat{K}) \frac{\partial \varepsilon}{\partial \bar{\theta}_{j}}=-i \varphi\left(\theta-\bar{\theta}_{j}-i \pi / 2\right), \quad j=1,2 \quad \text { and } \\
& (1-\hat{K}) \frac{\partial \varepsilon}{\partial \theta}=m L \sinh \theta+i \varphi\left(\theta-\bar{\theta}_{1}-i \pi / 2\right)+i \varphi\left(\theta-\bar{\theta}_{2}-i \pi / 2\right) .
\end{aligned}
$$



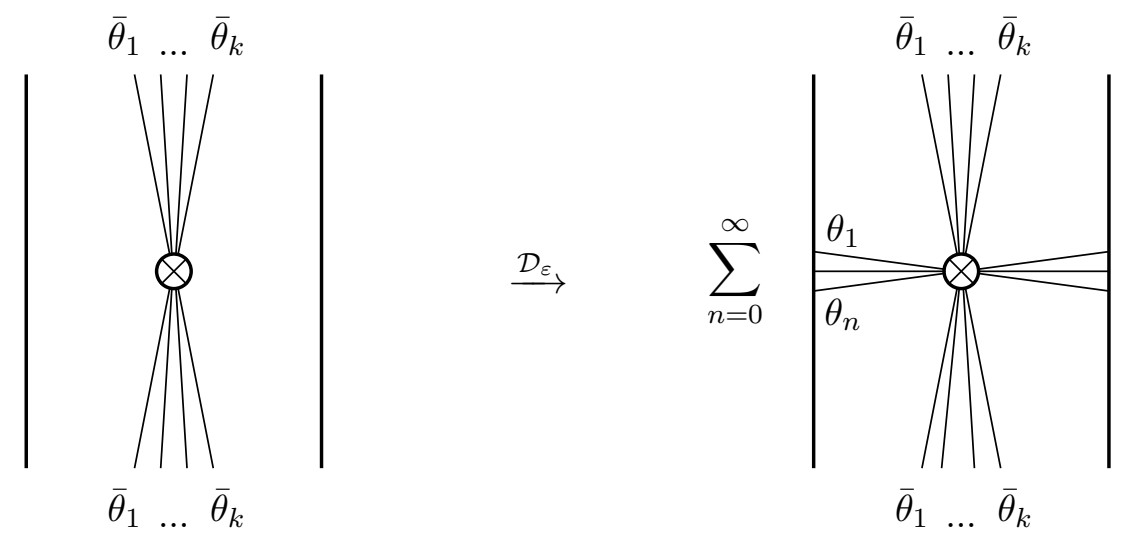

Figure 2: Graphical interpretation of the "dressing operation" $\mathcal{D}_{\varepsilon}$. Time runs in the vertical direction. In the horizontal direction periodic boundary conditions are understood.

Inverting the integral operator $\hat{K}$ and comparing with the explicit formulas (7.22)-(7.23) we find

$$
\mathcal{K}_{j k} \equiv \frac{\partial \bar{Q}_{j}}{\partial \bar{\theta}_{k}}=\left(\begin{array}{cc}
\mathcal{N}_{1}+\mathcal{N}_{\varphi} & -\mathcal{N}_{\varphi} \\
-\mathcal{N}_{\varphi} & \mathcal{N}_{2}+\mathcal{N}_{\varphi}
\end{array}\right)
$$

Therefore our final result (7.25) is indeed a "dressed version" of the asymptotic result (7.1), where instead of single form factors an appropriate integral series appears, and the derivatives of the asymptotic Bethe equations have been replaced by the total derivatives of the exact excited state quantization conditions.

\section{Conjecture for the general multiparticle case}

The results of the previous two sections suggest a simple generalization to the higher particle case. We formulate our conjecture below.

First it is useful to define a dressing function as follows. Given a certain diagonal form factor $F_{2 k}^{\mathcal{O}}\left(\bar{\theta}_{1}, \ldots, \bar{\theta}_{k}\right)$ its "dressed version" is defined as

$$
\begin{aligned}
\mathcal{D}_{\varepsilon}^{\mathcal{O}}\left(\bar{\theta}_{1}, \ldots, \bar{\theta}_{k}\right) \equiv \sum_{n=0}^{\infty} \frac{1}{n !} \int \frac{d \theta_{1}}{2 \pi} \ldots \frac{d \theta_{n}}{2 \pi}\left(\prod_{j} \frac{1}{1+e^{\varepsilon\left(\theta_{j}\right)}}\right) \\
F_{2(n+k), c}^{\mathcal{O}}\left(\bar{\theta}_{1}+i \pi / 2, \ldots, \bar{\theta}_{k}+i \pi / 2, \theta_{1}, \ldots, \theta_{n}\right) .
\end{aligned}
$$

The physical interpretation of this "dressing" is simply the addition of virtual particles which wind around the finite volume exactly one time. The imaginary shift of $i \pi / 2$ corresponds to the euclidean rotation of the particle world lines. A graphical interpretation of the dressing operation is shown in figure 2. The pseudoenergy $\varepsilon(\theta)$ is not defined at this stage, it is simply a parameter of the dressing operation.

Define the derivative matrix of the excited state quantization conditions and its determinant as

$$
\mathcal{K}_{j k}=\frac{\partial \bar{Q}_{j}}{\partial \bar{\theta}_{k}} \quad \bar{\rho}_{K}\left(\bar{\theta}_{1}, \ldots, \bar{\theta}_{K}\right)=\operatorname{det} \mathcal{K}_{j k} .
$$

For a given bipartite partition of the rapidities

$$
\begin{gathered}
\left\{\bar{\theta}_{1}, \ldots, \bar{\theta}_{K}\right\}=\left\{\bar{\theta}_{+}\right\} \cup\left\{\bar{\theta}_{-}\right\} \\
\left|\left\{\bar{\theta}_{+}\right\}\right|=K-n \text { and }\left|\left\{\bar{\theta}_{-}\right\}\right|=n
\end{gathered}
$$


define the restricted determinant

$$
\bar{\rho}_{K-n}\left(\left\{\bar{\theta}_{+}\right\} \mid\left\{\bar{\theta}_{-}\right\}\right)=\operatorname{det} \mathcal{K}_{+},
$$

where $\mathcal{K}_{+}$is the sub-matrix of $\mathcal{K}$ corresponding to the particles in the set $\left\{\bar{\theta}_{+}\right\}$.

With these notations, our conjectured expression for the exact finite volume mean values reads

$$
\begin{aligned}
{ }_{L}\left\langle\bar{\theta}_{1}, \ldots, \bar{\theta}_{K}|\mathcal{O}| \bar{\theta}_{1}, \ldots, \bar{\theta}_{K}\right\rangle_{L} & = \\
& \frac{1}{\bar{\rho}_{K}\left(\bar{\theta}_{1}, \ldots, \bar{\theta}_{K}\right)} \sum_{\left\{\bar{\theta}_{+}\right\} \cup\left\{\bar{\theta}_{-}\right\}} \mathcal{D}_{\varepsilon}^{\mathcal{O}}\left(\left\{\bar{\theta}_{-}\right\}\right) \bar{\rho}_{K-n}\left(\left\{\bar{\theta}_{+}\right\} \mid\left\{\bar{\theta}_{-}\right\}\right),
\end{aligned}
$$

where it is understood that the pseudoenergy $\varepsilon$ entering the dressing operation is the solution of the excited state TBA corresponding to the state $\left|\bar{\theta}_{1}, \ldots, \bar{\theta}_{K}\right\rangle_{L}$. The above equation is a generalization of the one-particle and two-particle results (6.15) and (7.25). Also, it can be regarded as the dressed version of the asymptotic formula (2.12).

\section{Conclusions}

In this paper we studied excited state mean values in finite volume integrable QFT. Although our ideas are general and should be applicable to arbitrary models with diagonal scattering, for technical reasons we restricted ourselves to theories with only one particle species. Moreover, we only considered the sinh-Gordon model because of the simplicity of its excited state TBA equations.

Our main results are (6.15) and (7.25) for the one-particle and two-particle mean values. In the previous section we also presented the formula (8.2) which is a conjectured generalization to arbitrary higher particle numbers.

Our calculations were based on two important conjectures. First of all, the basis for the present work is the LeClair-Mussardo integral series for the ground state mean values. Although this series is generally accepted to be true, a rigorous derivation from first principles is not yet known. The LM series was proven in [21] using the finite volume expansion (2.12). The present work shows that a derivation in the other direction is also possible: the result (2.12) follows from the LM series as a result of the analytic continuation and an appropriate summation procedure. It is interesting that neither the LM series nor the expansion (2.12) have been proven from first principles yet.

Our second assumption was that there is an analytic continuation procedure which connects a subset of the excited states to the ground state. This lead to the intermediate result (3.7). Although such an analytic continuation has been established for certain models, and it is believed to exist in other models as well, the general existence has not been proven. Moreover it is not known if it exists in the case of the sinh-Gordon model, which was our primary example.

In order to justify our results a number of checks can be performed. These include

- A careful investigation of the IR limit. In the sinh-Gordon model we calculated the first exponential corrections of order $e^{-m R}$ by an independent method and found agreement with the first terms of the IR expansion of (6.15) and (7.25). This calculation will be presented elsewhere.

- Considering the trace of the energy-momentum tensor: $\mathcal{O}=\Theta=T_{\mu}^{\mu}$. In this case there is an exact relation

$$
\langle n|\Theta| n\rangle_{L}=\frac{E_{n}}{L}+\frac{d E_{n}}{d L}
$$

valid for arbitrary finite volume states. The right hand side of this equation can be evaluated using the known excited state TBA. On the other hand, the l.h.s. can also be evaluated using 
our integral series and the known form factors of $\Theta[18 \|$. We performed this comparison and found an exact agreement. This provides an important consistency check of our calculations. The calculation itself is a simple generalization of the corresponding calculation presented in [18] for the ground state mean value.

- The investigation of the UV limit. Calculating the $m L \rightarrow 0$ limit of all the integrals in the infinite series and summing up the leading contributions it should be possible to recover matrix elements calculated in the conformal limit. We leave this problem to further research.

The technical details of the calculation show that once it is established how to represent individual particles in the excited state TBA then the rule to get the mean values is to substitute the complex rapidities into formulas of the form (8.2). One-particle states are typically represented as complex conjugate pairs of rapidities, possibly of a particle of different type. In these cases the complex conjugate pairs have to be substituted into (8.2); this way a one-particle mean value will be given by our two-particle formula. This is in complete accordance with the findings of [30] which used essentially the same idea to obtain the leading exponential correction, the so-called $\mu$-term. The study of such cases will be pursued in a future publication.

We find it remarkable that our final results for the one-particle and two-particle mean values (eqs. (6.15) and (7.25)) are relatively simple and intuitive compared to the cumbersome way in which they were derived from the starting point (3.7). Also, it is quite remarkable that the normalization factor in the final formula is simply the total derivative (or Jacobian) of the exact quantization condition for the Bethe roots. This calls for an alternative derivation of the present results.

\section{Acknowledgements}

We would like to thank Gábor Takács for numerous discussions, for suggesting the proof of Theorem 1 and for many useful comments on the manuscript. Also, the author is grateful to Patrick Dorey and Roberto Tateo for a stimulating discussion at a conference in Bologna in 2011.

Most of this work was carried out while the author was employed by the NWO/VENI grant 016.119.023 at the University of Amsterdam, the Netherlands.

\section{References}

[1] M. Luscher, "Volume Dependence of the Energy Spectrum in Massive Quantum Field Theories. 1. Stable Particle States," Commun.Math.Phys. 104 (1986) 177.

[2] M. Luscher, "Volume Dependence of the Energy Spectrum in Massive Quantum Field Theories. 2. Scattering States," Commun.Math.Phys. 105 (1986) 153-188.

[3] L. Lellouch and M. Luscher, "Weak transition matrix elements from finite volume correlation functions," Commun.Math.Phys. 219 (2001) 31-44, arXiv:hep-lat/0003023 [hep-lat].

[4] Al. B. Zamolodchikov, "Thermodynamic Bethe Ansatz in relativistic models. Scaling three state Potts and Lee-Yang models," Nucl. Phys. B342 (1990) 695-720.

[5] T. R. Klassen and E. Melzer, "Purely Elastic Scattering Theories and their Ultraviolet Limits," Nucl. Phys. B338 (1990) 485-528.

[6] C. Destri and H. J. de Vega, "New thermodynamic Bethe ansatz equations without strings," Phys. Rev. Lett. 69 (1992) no. 16, 2313-2317.

[7] C. Destri and H. J. de Vega, "Unified approach to Thermodynamic Bethe Ansatz and finite size corrections for lattice models and field theories," Nucl. Phys. B 438 (1995) no. $3,413-454$. 
[8] V. V. Bazhanov, S. L. Lukyanov, and A. B. Zamolodchikov, "Integrable structure of conformal field theory. 2. Q operator and DDV equation," Commun.Math.Phys. 190 (1997) 247-278, arXiv:hep-th/9604044 [hep-th].

[9] P. Dorey and R. Tateo, "Excited states by analytic continuation of TBA equations," Nucl.Phys. B482 (1996) 639-659, arXiv:hep-th/9607167 [hep-th].

[10] P. Dorey and R. Tateo, "Excited states in some simple perturbed conformal field theories," Nucl.Phys. B515 (1998) 575-623, arXiv:hep-th/9706140 [hep-th].

[11] C. Destri and H. de Vega, "Nonlinear integral equation and excited states scaling functions in the sine-Gordon model," Nucl.Phys. B504 (1997) 621-664, arXiv:hep-th/9701107 [hep-th].

[12] G. Feverati, F. Ravanini, and G. Takacs, "Nonlinear integral equation and finite volume spectrum of Sine-Gordon theory," Nucl.Phys. B540 (1999) 543-586,

arXiv:hep-th/9805117 [hep-th].

[13] G. Feverati, F. Ravanini, and G. Takács, "Scaling functions in the odd charge sector of sine-Gordon/massive Thirring theory," Physics Letters B 444 (1998) 442-450, arXiv:hep-th/9807160.

[14] V. V. Bazhanov, S. L. Lukyanov, and A. B. Zamolodchikov, "Integrable quantum field theories in finite volume: Excited state energies," Nucl.Phys. B489 (1997) 487-531, arXiv:hep-th/9607099 [hep-th].

[15] N. Beisert, C. Ahn, L. F. Alday, Z. Bajnok, J. M. Drummond, et al., "Review of AdS/CFT Integrability: An Overview," Lett.Math.Phys. 99 (2012) 3-32, arXiv:1012.3982 [hep-th].

[16] F. H. L. Essler and R. M. Konik, "Applications of Massive Integrable Quantum Field Theories to Problems in Condensed Matter Physics," eprint arXiv:cond-mat/0412421 (2004), arXiv:cond-mat/0412421.

[17] A. Leclair, F. Lesage, S. Sachdev, and H. Saleur, "Finite temperature correlations in the one-dimensional quantum Ising model," Nucl. Phys. B482 (1996) 579-612, arXiv: cond-mat/9606104.

[18] A. Leclair and G. Mussardo, "Finite temperature correlation functions in integrable QFT," Nucl. Phys. B552 (1999) 624-642, arXiv:hep-th/9902075.

[19] B. Doyon, "Finite-temperature form-factors: A Review," SIGMA 3 (2007) 011, arXiv:hep-th/0611066 [hep-th].

[20] B. Pozsgay and G. Takacs, "Form factors in finite volume II:disconnected terms and finite temperature correlators," Nucl. Phys. B788 (2008) 209-251, arXiv:0706.3605 [hep-th].

[21] B. Pozsgay, "Mean values of local operators in highly excited Bethe states," J. Stat. Mech. 2011 (2011) P01011, arXiv:1009.4662 [hep-th].

[22] F. H. L. Essler and R. M. Konik, "Finite-temperature dynamical correlations in massive integrable quantum field theories," J. Stat. Mech. 0909 (2009) P09018, arXiv:0907.0779 [cond-mat.str-el].

[23] H. Saleur, "A comment on finite temperature correlations in integrable QFT," Nucl. Phys. B567 (2000) 602-610, arXiv:hep-th/9909019.

[24] I. Szecsenyi, G. Takacs, and G. Watts, "One-point functions in finite volume/temperature: a case study," arXiv:1304.3275 [hep-th].

[25] O. A. Castro-Alvaredo and A. Fring, "Finite temperature correlation functions from form factors," Nucl. Phys. B636 (2002) 611-631, arXiv:hep-th/0203130.

[26] B. Pozsgay and G. Takacs, "Form factor expansion for thermal correlators," J. Stat. Mech. $11(2010)$ 12, arXiv:1008.3810 [hep-th]. 
[27] B. Pozsgay, "Finite volume form factors and correlation functions at finite temperature," arXiv:0907.4306 [hep-th].

[28] I. Szecsenyi and G. Takacs, "Spectral expansion for finite temperature two-point functions and clustering," J.Stat.Mech. 1212 (2012) P12002, arXiv:1210.0331 [hep-th].

[29] B. Pozsgay and G. Takacs, "Form factors in finite volume I: form factor bootstrap and truncated conformal space," Nucl. Phys. B788 (2008) 167-208, arXiv:0706.1445 [hep-th].

[30] B. Pozsgay, "Luscher's mu-term and finite volume bootstrap principle for scattering states and form factors," Nucl. Phys. B802 (2008) 435-457, arXiv:0803.4445 [hep-th].

[31] G. Takacs, "Determining matrix elements and resonance widths from finite volume: The Dangerous $\mu$-terms," JHEP 1111 (2011) 113, arXiv:1110.2181 [hep-th].

[32] F. A. Smirnov, "Form-factors in completely integrable models of quantum field theory," Adv. Ser. Math. Phys. 14 (1992) 1-208.

[33] H. M. Babujian, A. Foerster, and M. Karowski, "The form factor program: A review and new results - the nested SU(N) off-shell Bethe ansatz," SIGMA 2 (2006) 082, arXiv:hep-th/0609130.

[34] M. Gaudin, "La function d'onde de Bethe pour les modèles exacts de la mécanique statistique," Commisariat á l'énergie atomique, Paris (1983).

[35] V. Korepin, N. Bogoliubov, and A. Izergin, Quantum inverse scattering method and correlation functions. Cambridge University Press, 1993.

[36] T. R. Klassen and E. Melzer, "On the relation between scattering amplitudes and finite size mass corrections in QFT," Nucl. Phys. B362 (1991) 329-388.

[37] T. Palmai and G. Takacs, "Diagonal multi-soliton matrix elements in finite volume," Phys.Rev. D87 (2013) 045010, arXiv:1209.6034 [hep-th].

[38] C. M. Bender and T. T. Wu, "Anharmonic Oscillator," Phys. Rev. 184 (1969) 1231-1260.

[39] J. Teschner, "On the spectrum of the Sinh-Gordon model in finite volume," Nucl. Phys. B799 (2008) 403-429, arXiv:hep-th/0702214.

[40] A. Klümper and P. A. Pearce, "Analytic calculation of scaling dimensions: Tricritical hard squares and critical hard hexagons," Journal of Statistical Physics 64 (1991) 13-76.

[41] A. Klümper and P. A. Pearce, "Conformal weights of RSOS lattice models and their fusion hierarchies," Physica A Statistical Mechanics and its Applications 183 (1992) 304-350.

[42] G. Delfino, P. Simonetti, and J. L. Cardy, "Asymptotic factorisation of form factors in two-dimensional quantum field theory," Phys. Lett. B387 (1996) 327-333,

arXiv:hep-th/9607046. 